\title{
CT-gestützte Drahtmarkierung vor videoassistierter thorakoskopischer OP von pulmonalen Rundherden - eine Auswertung von 184 Fällen
}

\author{
Dissertation \\ zur Erlangung des akademischen Grades \\ Dr. med.
}

an der Medizinischen Fakultät

der Universität Leipzig

eingereicht von: Marie-Kristin Schulze

geboren am: $\quad$ 05.01.1988 in Bautzen

angefertigt am / in: Universität Leipzig / Medizinische Fakultät

Klinik und Poliklinik für Diagnostische und Interventionelle Radiologie

Betreuer: $\quad$ Prof. Dr. med. Thomas Kahn

OA Dr. med. Patrick Stumpp,

Klinik und Poliklinik für Diagnostische und Interventionelle Radiologie 


\section{Inhaltsverzeichnis}

1. Bibliographische Zusammenfassung 1

2. Einführung 2

2.1. Definition des Begriffs „pulmonaler Rundherd“ 2

2.2. Diagnostik pulmonaler Rundherde 3

2.3. Resektionsverfahren pulmonaler Rundherde 4

2.3.1. Offene Thorakotomie 4

2.3.2. Videoassistierte thorakoskopische Chirurgie (VATS) 5

2.3.2.1. Geschichte der VATS 5

2.3.2.2. Indikationen und Ablauf der VATS 6

$\begin{array}{ll}\text { 2.3.2.3. Vor- und Nachteile der VATS } & 7\end{array}$

2.4. Präoperative Markierungstechniken vor der VATS 8

2.4.1. Übersicht der verschiedenen Markierungstechniken 8

2.4.1.1. Die Farbmarkierung 9

2.4.1.2. Präoperative Markierung mit Lipiodol 10

2.4.1.3. Präoperative Markierung mit Radionukleotiden $\quad 10$

2.4.2. Präoperative Drahtmarkierung 11

2.4.2.1. Präoperative Markierung mit dem Lungen-Marker- $\quad 11$ System der Firma Somatex ${ }^{\circledast}$

2.5. Zielsetzung 13

3. Publikation 14

4. Zusammenfassung 22

5. Literaturverzeichnis 27

6. Erklärung über die eigenständige Abfassung der Arbeit 33

7. Lebenslauf 34

8. Danksagung 36 


\section{Bibliographische Beschreibung:}

Name: Marie-Kristin Schulze

Titel: CT-gestützte Drahtmarkierung vor videoassistierter thorakoskopischer OP von pulmonalen Rundherden - eine Auswertung von 184 Fällen

Universität Leipzig, Publikationspromotion

36 S., 62 Lit., 5 Abb., 5 Tab.

\section{Referat:}

Die videoassistierte thorakoskopische Chirurgie (VATS - video-assisted thoracoscopic surgery) ist heutzutage als minimal-invasive Operation in der Diagnostik und Therapie von suspekten Lungenrundherden fest etabliert. Vor allem für die Resektion kleiner, peripher gelegener Rundherde unklarer Dignität stellt die VATS derzeit das Verfahren der Wahl dar. Im Vergleich zur offenen Thorakotomie hat dieser minimal-invasive Eingriff bedeutsame Vorteile in Bezug auf Morbidität, Mortalität und Rekonvaleszenz. Ein wesentlicher Nachteil besteht in der fehlenden Möglichkeit der digitalen Palpation des Herdes während der thorakoskopischen Exploration der Lunge. Um dennoch kleine, subpleural gelegene Rundherde sicher lokalisieren und somit resezieren zu können, wurden diverse präoperative Markierungstechniken entwickelt. Beispielsweise wurden Hakendrähte, Methylenblau zur Anfärbung der Herde, Radionucleotide und Lipiodol genutzt. In unserem Haus wird seit circa zehn Jahren ein speziell für diesen Zweck entwickelter Spiraldraht (Somatex Medical Technologies $\mathrm{GmbH}$, Berlin, Deutschland) verwendet, welcher CT-gestützt unmittelbar präoperativ an den Herd gebracht wird.

Die Zielsetzung dieser Arbeit bestand in der Beurteilung der Wertigkeit der präoperativen CT-gestützten Drahtmarkierung von kleinen, peripher gelegenen Rundherden bei der Verwendung des Lungen-Marker-Systems der Firma Somatex ${ }^{\circledR}$ vor videothorakoskopischer Resektion. Wir berichten über unsere Erfahrung mit diesem speziellen System bei 184 Markierungen in dem Zeitraum von Oktober 1999 bis März 2010. 


\section{Einführung}

\subsection{Definition des Begriffs „pulmonaler Rundherd“}

Der Begriff „solitärer pulmonaler Rundherd“ ist definiert als eine annähernd runde bzw. kugelförmige Läsion, welche vollständig von Lungengewebe umgeben ist, einen Durchmesser von $3 \mathrm{~cm}$ nicht überschreitet und keine Umgebungsauffälligkeiten wie Atelektasen oder Adenopathien aufweist (Ost et al. 2003). Ein Herd mit einem Durchmesser von mehr als $3 \mathrm{~cm}$ wird eher als „pulmonale Raumforderung“ (engl.: „pulmonary mass“, „lung mass") bezeichnet und erweist sich in der histologischen Untersuchung häufig als maligne (Tan et al. 2003). Aus früheren groß angelegten Screening-Untersuchungen ist bekannt, dass pulmonale Rundherde in 0,09 bis 0,2\% aller durchgeführten RöntgenThoraxaufnahmen diagnostiziert werden, d.h. circa 150000 dieser Herde werden jährlich in den Vereinigten Staaten entdeckt (Ost et al. 2003). Die Mehrzahl der gefundenen Herde treten dabei als Zufallsbefunde auf. Durch die ständige Verbesserung und Weiterentwicklung bildgebender Verfahren, wie der Dünnschicht-Spiral-CT, ist die Zahl neu entdeckter Rundherde in den letzten Jahren weiter gestiegen (Fischbach et al. 2003, Shiau et al. 2007). Im Durchschnitt sind circa 50\% aller neu entdeckten Rundherde maligne (Mack et al. 1993, Hoffmann et al. 2000). Da das Bronchialkarzinom eine Mortalitätsrate von bis zu 85\% aufweist, steht die Sicherung der Dignität eines Rundherdes an oberster Stelle. Die Differentialdiagnose der benignen Herde umfasst im Wesentlichen entzündliche und infektiöse Geschehen (z.B. Bakterien, Pilze, Wurminfektionen), autoimmunologische Erkrankungen (z.B. Rheumaknoten, Mb. Wegener, Sarkoidose), angeboren-degenerative Ursachen (z.B. bronchogene Zyste) und benigne Tumore wie Lipome, Fibrome oder Chondrome. Auch intrapulmonale Lymphknoten oder traumatisch bedingte Narben lassen sich hier nennen.

Bei den malignen Befunden unterscheidet man zwischen primären Lungenkarzinomen (v. a. Adeno- und Plattenepithelkarzinome) und Metastasen. In seltenen Fällen können sich auch Sarkome, Lymphome oder Teratome hinter einem pulmonalen Rundherd verbergen.

Der häufig als Zufallsbefund entdeckte pulmonale Rundherd stellt den behandelnden Mediziner immer vor die Frage der Dignität - benigne versus maligne. Die meisten Rundherde sind asymptomatisch und dennoch erweisen sich circa 50\% als maligne (Lillington et al. 1993). Vor allem Lungenkarzinome in sehr frühen Stadien der Erkrankung können sich 
als pulmonaler Rundherd darstellen. Somit muss ein neu diagnostizierter, radiologisch erfasster Rundherd immer als malignitätsverdächtig gelten, bis nicht eindeutig das Gegenteil erwiesen ist. Die zeitnahe chirurgische Entfernung eines malignen Herdes ist von entscheidender Bedeutung, da die Prognose des Lungenkarzinoms vom Stadium bei Diagnosestellung abhängt (Suzuki et al 1999). Es lässt sich jedoch mit keinem nicht-invasiven diagnostischen Verfahren die Dignität sicher bestimmen.

\subsection{Diagnostik pulmonaler Rundherde}

Die Differentialdiagnose gutartig versus bösartig wird vorrangig von vier Einflussfaktoren bestimmt: Der Größe des pulmonalen Rundherdes, das Alter des Patienten, Nikotinabusus in der Vorgeschichte sowie radiologische Charakteristika des Herdes. Eher für ein benignes Geschehen sprechen bildmorphologisch u.a. : Eine kleine Herdgröße, eine glatte Begrenzung, Kalzifizierungen oder Fetteinlagerungen im Herd, Dichteanhebungen im Kontrastmittel-CT von weniger als 15 Hounsfield-Einheiten sowie eine Größenstabilität in bildgebenden Verfahren über zwei Jahre. Demgegenüber sind größere Herde mit einer unscharfen Randbegrenzung, eventuell mit der Ausprägung von Spikulae, eher als malignitätsverdächtig anzusehen (Siegelman et al. 1986, Gurney et al. 1993). Die Wahrscheinlichkeit für das Vorliegen von Malignität steigt ebenfalls mit zunehmendem Patientenalter, einer Karzinomerkrankung in der Vorgeschichte und bekanntem Nikotinabusus. Auch wenn all diese Kriterien wichtige Hinweise für die Charakterisierung der gefundenen pulmonalen Rundherde geben, lässt sich in den meisten Fällen ein invasives Vorgehen mit der Sicherung einer diagnosebringenden Zyto- und Histologie kaum umgehen. Die diagnostische Ausbeute zytologischer Verfahren hängt in großem Maße von der Tumorlokalisation der Bronchialkarzinome ab: Je zentraler ein Tumor lokalisiert ist, umso höher ist die diagnostische Sicherheit. Verglichen mit der Histologie ist besonders bei der zytologischen Tumorklassifikation mit einer Einschränkung der Sicherheit zu rechnen (Gatzemeier 2002). Nicht selten werden nicht genügend oder für die Veränderung nicht repräsentative Zellen

gewonnen. Des Weiteren lässt sich weder die Ausbreitung des Tumors, das Wachstumsmuster noch die Architektur des Gewebes zytologisch direkt erfassen. 
Je nach Größe und Lage des Herdes stehen derzeit verschiedene Verfahren zur Biopsiegewinnung zur Verfügung. Größere zentral gelegene Herde können mit der transbronchialen Biopsie im Rahmen einer Bronchoskopie gut erreicht werden. Die transthorakale CT-gestützte Punktion eignet sich zur Sicherung peripherer Rundherde ab einer Größe von ca. $1 \mathrm{~cm}$. Kleine Herde lassen sich mit dieser Methode jedoch nur schwer erreichen und erlauben in der Mehrzahl der Fälle nur eine eingeschränkte Aussagekraft der Punktion (Thomas 2005). Methode der Wahl zur histologischen Sicherung kleiner, peripherer Rundherde stellt derzeit die videothorakoskopische Resektion dar. Sowohl im diagnostischen als auch im therapeutischen Bereich hat diese die offene Thorakotomie heutzutage weitestgehend abgelöst. Der Erfolg dieser Methode wird durch eine präoperative Lokalisation mittels Drahtmarkierung noch erhöht.

\subsection{Resektionsverfahren pulmonaler Rundherde}

\subsubsection{Offene Thorakotomie}

Die operative Eröffnung des Brustkorbs stellte die Mediziner lange Zeit vor große Schwierigkeiten. Erst durch die Einführung eines Verfahrens, welches von Ferdinand Sauerbruch 1904 entwickelt wurde, hielt die Thoraxchirurgie Einzug in die Medizin. Er konstruierte eine große Unterdruckkammer, in der nur circa ein Zehntel des normalen Luftdruckes herrschte. Somit war die Eröffnung des Brustkorbes ohne die Gefahr der Entwicklung eines tödlichen Pneumothorax erstmals möglich. Später wurde das Verfahren dahingehend geändert, dass nicht von außen ein Unterdruck erzeugt, sondern die Lunge von innen mit einem höheren Druck stabilisiert wurde (die sogenannte Überdruckbeatmung). Mittels dieser neuen Methoden war der chirurgische Weg zu den Thoraxorganen nun möglich.

Die Art des Eingriffs bestimmt bei der Thorakotomie die Schnittführung. Während fast alle Operationen am offenen Herzen über eine Sternotomie erfolgen, bieten sich für Eingriffe an Mediastinum und Lunge eher laterale Zugänge an. Dabei unterscheidet man die anterolaterale von der posterolateralen Thorakotomie. Meist wird die Pleurahöhle dabei im 5. Interkostalraum eröffnet. Ein Vorteil der antero- gegenüber der posterolateralen Schnittführung liegt darin, dass weniger Muskulatur zerstört wird, da lediglich der $\mathrm{M}$. 
serratus von der Rippe abgelöst wird. Demgegenüber steht die komplette Durchtrennung des M. latissimus bei der posteroleteralen Thorakotomie. Um postoperativ auftretende Restblutungen, Sekret und Luft zu drainieren, werden nach jeder Thorakotomie ein bis zwei Drainagen in die Pleurahöhle eingelegt. In der Regel können diese am 2. bis 5. postoperativen Tag gezogen werden.

Indikationen für eine offene Thorakotomie stellen u.a. großflächige Lungenresektionen und Lobektomien bis hin zur Pneumektomie dar, d.h. die Entfernung eines ganzen Lungenflügels bei z.B. zentral sitzenden Karzinomen. Auch ausgedehnte Pleuraverwachsungen können einen videothorakoskopischen Eingriff unmöglich machen.

Postoperativ besteht die Gefahr in der Entwicklung respiratorischer oder kardialer Insuffizienzen. Weitere postoperative Komplikationen betreffen vor allem Nachblutungen und Auftreten von Pneumothoraces.

\subsubsection{Videoassistierte thorakoskopische Chirurgie (VATS)}

\subsubsection{Geschichte der VATS}

Der Grundstein der Thorakoskopie wurde 1910 von dem schwedischen Internisten HansChristian Jacobaeus (1879 - 1937) mit der Einführung des Thorakoskops gelegt. Grundlage dafür stellte das kurz zuvor entwickelte Zystoskop dar. In dem Artikel „Über die Möglichkeit, die Zystoskopie bei Untersuchungen seröser Höhlungen anzuwenden“ (Jacobaeus 1910) veröffentlichte Jacobaeus seine ersten Erfahrungen. Bereits 1925 erschien eine ausgedehnte Zusammenfassung seiner bisherigen Arbeit. In über einhundert Fällen hatte er Pleuraergüsse oder Tumore mittels dieser Methode diagnostizieren können (Jacobaeus 1925). Anfangs nur für diagnostische Zwecke genutzt, gewann die Thorakoskopie in den folgenden Jahren auch immer mehr an therapeutischer Bedeutung. Vor allem die Beseitigung pleuraler Adhäsionen im Zusammenhang mit der damaligen Tuberkulosetherapie rückte ins Zentrum des therapeutischen Interesses. Als zwischen 1950 und 1960 die Tuberkulostatikatherapie in die Tuberkulosebehandlung eingeführt wurde, rückte die Thorakoskopie zunächst wieder in den Hintergrund und wurde lediglich noch zur Diagnostik und nicht mehr zu therapeutischen Zwecken angewandt (Braimbridge 1993). Neuen Aufschwung erhielt sie mit der Einführung der Videotechnik. Der Kieler Gynäkologe Kurt Semm (1927 - 2003) führte 1978 die laparoskopische Chirurgie in der Frauenheilkunde ein. Vier Jahre darauf führte er die erste 
laparoskopische Appendektomie durch (Semm 1983). Ein weiterer großer Durchbruch gelang dem deutschen Chirurgen Erich Mühe (1938 - 2005) im Jahr 1985 mit der Durchführung der ersten laparoskopischen Cholecystektomie, auch wenn dieser Verdienst in der Literatur oft fälschlicherweise dem Franzosen Phillipe Mouret angerechnet wird (Reynolds 2001). Mit diesen Erfolgen und der Einführung videooptischer Techniken rückte die endoskopische Chirurgie in den Mittelpunkt des Interesses vieler Mediziner und hielt nun auch Einzug in die Thoraxchirurgie. Die verbesserte optische Auflösung der endoskopischen Teleskope und eine Vielzahl neuer endoskopischer Instrumente wie Zangen, Stapler oder Skalpelle ermöglichten das Voranschreiten der Thorakoskopie. Dank der Einführung modernster Videokameras hatte nun nicht nur der Operateur allein Einblick in den OP-Situs. Dem gesamten Operationsteam war es nun möglich einzelne Operationsschritte auf Bildschirmen mitzuverfolgen. In thoraxchirurgischen Zentren werden heutzutage nahezu 30\% aller thoraxchirurgischen Eingriffe mittels der videoassistierten Thorakoskopie durchgeführt (Rieger et al. 1997).

\subsubsection{Indikationen und Ablauf der VATS}

Dank der Entwicklung zahlreicher technischer Neuerungen in den letzten Jahren hat sich das Einsatzspektrum der videoassistierten Thoraxchirurgie erheblich erweitert und sie kann nun sowohl zu diagnostischen wie auch therapeutischen Zwecken im Thoraxraum genutzt werden. Neben parenchymatösen Lungenerkrankungen stellen pleurale und mediastinale Pathologien sowie Eingriffe am Perikard Hauptzielgebiete der VATS dar.

Die diagnostisch angewendete VATS wird vor allem zur Histologie- und Ergussgewinnung mit anschließender Probenanalyse genutzt. Neben der Resektion unklarer pulmonaler Lungenrundherde, werden u.a. Biopsien der Pleura, Lymphknoten oder mediastinaler Tumore, sowie Probeexzisionen des Lungenparenchyms zur Diagnosesicherung interstitieller Lungenkrankheiten durchgeführt (Eckersberger 1997, Solli et al. 2007).

In gleicher Weise hat auch das therapeutische Spektrum der VATS in den letzten Jahren stetig zugenommen. Für die Behandlung des sekundären Pneumothorax stellte sich die thorakoskopische Pleurodese verglichen mit einer ausschließlichen Drainagebehandlung bezüglich der Rezidivrate als vorteilhaft heraus (Pirker 2005, Kim et al. 2011). Aber auch 
anatomische und atypische Lungenresektionen, die Entfernung mediastinaler Tumore, die Behandlung von Pleuraempyemen oder das Anlegen von Pleurodesen bei Ergüssen malignen (Dohrmann et al. 2001) oder benignen Ursprungs zählen zum therapeutischen Einsatzgebiet der Thorakoskopie (Kaiser 1994, Eckersberger 1997, Solli et al. 2007). Für eine exakte Planung des gesamten thorakoskopischen Eingriffs ist eine präoperative Bildgebung erforderlich, welche in der Regel mit einer Computertomographie erfolgt. Die videoassistierte Thorakoskopie wird in Allgemeinnarkose und Doppellumenintubation durchgeführt, womit eine seitengetrennte und einseitige Ventilation ermöglicht wird. Nach der Seitenlagerung des Patienten, Hautdesinfektion und sterilen Abdeckung des Operationsgebietes erfolgt die Platzierung der einzelnen Trokare. Der Abstand der Trokarhülsen zueinander sollte groß genug gewählt werden, um eine gegenseitige Behinderung der Instrumente und der Optik intraoperativ zu vermeiden. Vor dem Beginn der eigentlichen Operation ist eine visuell-endoskopische Exploration der Thoraxhöhle Pflicht. Die Resektion verschiedenster Pathologien von Lunge, Pleura oder auch Mediastinum wurde erst durch die Entwicklung spezieller endoskopischer Instrumente möglich. Endoskopische Schneidegeräte, Fass- und Clipzangen sowie sogenannte Endostapler, welche Parenchymdurchtrennung und gleichzeitigen Parenchymverschluss in einem Arbeitsschritt gewährleisten, erlauben heutzutage den immer weiträumigeren Einsatz der VATS in der Thoraxchirurgie (Inderbitzi et al. 1993).

Nach Abschluss der Operation und Kontrolle auf Luftdichtigkeit und Blutstillung schließt sich die Einlage von ein bis zwei Thoraxdrainagen an.

Als postoperative Komplikationen können Pneumothoraces, Pleuraempyeme, Hämatothoraces oder Pneumonien infolge postoperativer Sekretretention auftreten. Schwerwiegende Komplikationen, wie großflächige intrathorakale Blutungen, finden sich bei der videoassistierten Thorakoskopie selten.

\subsubsection{Vor- und Nachteile der VATS}

Die Vorteile minimal-invasiver Eingriffe gegenüber offenen Operationsverfahren sind unbestreitbar. Durch die Nutzung sehr kleiner, nur circa 1,5 cm langer Inzisionen und die damit verringerte Traumatisierung des Brustkorbes resultiert eine geringere Morbidität und Mortalität der VATS gegenüber der offenen Thorakotomie. Durch die dezimierte 
Verletzungsgefahr umgebender Strukturen und die kleineren Wundflächen kommt es zu einer geringeren postoperativen Schmerzbelastung des Patienten und somit zu einem geringeren Schmerzmittelverbrauch (Landreneau et al. 1993). Die reduzierte Schmerzbelastung führt wiederum zu einem frühen Erreichen der individuellen Lungenfunktion und einer zügigeren Rekonvaleszenz des Patienten, verbunden mit einer kürzeren Krankenhausaufenthaltsdauer (Sedrakyan et al. 2004). Das Risiko der bei offenen Thorakotomien gefürchteten Wundinfektionen konnte durch die kleinen Schnittflächen ebenfalls reduziert werden. Auch das kosmetisch bessere Ergebnis durch die Entstehung nur weniger Zentimeter großer Narben lässt sich hier nennen. Durch das verminderte Zugangstrauma entstehen postoperativ weniger Adhäsionen im Operationsgebiet, was spätere Reoperationen erleichtert.

Eine Einschränkung besteht in der Bedingung der relativ peripheren Lage des zu resezierenden Lungenparenchyms. Eine sichere videoassistierte Resektion, beispielsweise eines suspekten Lungenrundherdes, ist nur möglich, wenn sich dieser in relativer Nähe zur Pleura befindet. Auch die fehlende manuelle Palpationsmöglichkeit während der Operation, vor allem bei der Resektion maligner Befunde und dem Metastasenausschluss von Bedeutung, stellt einen Nachteil der VATS dar.

Die Komplikationsrate bei videoassistierten Eingriffen ist gering und steigt mit zunehmendem Patientenalter und der Größe des zu resezierenden Herdes an (Jiménez et al. 2001). Intraoperativ stellen vor allem Blutungen Risiken dar, welche unter Umständen ein Umsteigen auf eine offene Thorakotomie erfordern können, sollten diese nicht endoskopisch beherrschbar sein. Die persistierende Luftfistel ist die häufigste postoperative Komplikation, gefolgt von Pneumonien und Wundinfektionen (Yim et al. 1996). Eine gefürchtete, aber glücklicherweise seltene Komplikation bei der Bergung maligner Tumore stellen die Impfmetastasen dar.

\subsection{Präoperative Markierungstechniken vor der VATS}

\subsection{1. Übersicht der verschiedenen Markierungstechniken}

Vor allem die Detektion sehr kleiner $(<1 \mathrm{~cm})$ Lungenrundherde kann sich intraoperativ, aufgrund der fehlenden Möglichkeit der manuellen Palpation während der 
Videothorakoskopie, oft schwierig darstellen. Auch die Orientierung anhand topographischer Landmarken geht durch den iatrogen erzeugten Lungenkollaps während der Prozedur verloren. Um eine bessere intraoperative Lokalisation zu ermöglichen, wurden aufgrund dessen vielfältige präoperative Markierungstechniken entwickelt, auf welche im Folgenden kurz eingegangen werden soll.

\subsubsection{Die Farbmarkierung}

Die präoperative Farbmarkierung erfolgt unmittelbar vor der VATS. Dabei wird der betreffende Herd in Lokalanästhesie CT-gestützt mit Hilfe einer 22 bzw. 25-Gauge-Nadel punktiert. Anschließend wird Methylenblau in oder möglichst nah am Rundherd, sowie während des Rückzugs der Nadel, injiziert. Somit ist auch die Markierung des Nadelverlaufs und der visceralen und parietalen Pleura gewährleistet. Die mittlere Interventionsdauer beträgt dabei $15-47$ min. Als Komplikationen werden Pneumothoraces und intrapulmonale Blutungen beschrieben (Wicky et al. 1994, Lenglinger et al. 1994). Shah und Kollegen beschreiben die Entwicklung eines Schockzustandes bei einem Patienten kurz nach der Injektion von Methylenblau (Shah et al. 1993). Als ungünstig erwies sich bei der Verwendung von gefärbten Substanzen das Auslaufen in umliegendes Gewebe und somit die Maskierung des Herdes. Darüber hinaus ist die Färbung mit Methylenblau eine relativ kurzlebige Methode, da die Farbe nur für einen kurzen Zeitraum sichtbar bleibt und die VATS innerhalb von circa drei Stunden erfolgen muss (Lenglinger et al.1994).

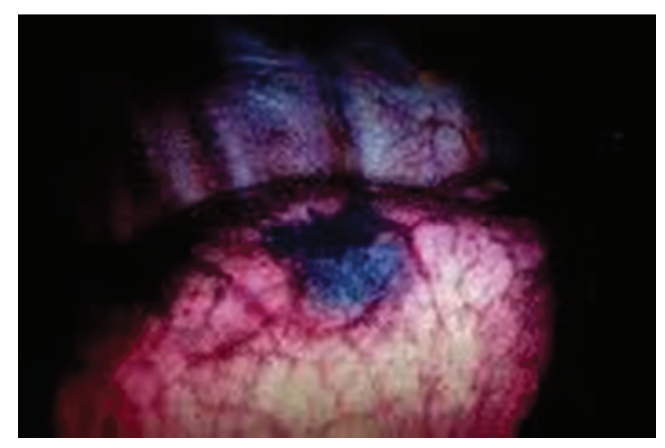

Pulmonaler Rundherd nach präoperativer Markierung mit Methylenblau

(Von: Lin J, lannettoni M. The Role of Video-assisted Thoracic Surgery in the Management of Lung Cancer. Section of General Thoracic Surgery, Department of Surgery University of Michigan Medical Center. CancerNews) 


\subsubsection{Präoperative Markierung mit Lipiodol}

Lipiodol ist ein nicht wasserlösliches, iodhaltiges Röntgenkontrastmittel, welches üblicherweise zur röntgenologischen Darstellung der Lymphgefäße und Lymphknoten (Lymphographie) genutzt wird. Ein weiteres Einsatzgebiet stellt derzeit die präoperative Markierung vor allem sehr kleiner, tiefliegender Rundherde vor der Thorakoskopie dar. In einem Zeitraum von einem bis sieben Tage vor dem thorakoskopischen Eingriff werden die Herde CT-gestützt mit Lipiodol markiert. Nach erfolgter Lokalanästhesie werden mit Hilfe einer 22- bzw. 23-Gauge-Nadel circa 0,3-0,5 ml des Kontrastmittels in bzw. am Rundherd appliziert. Intraoperativ sind die markierten Herde unter Durchleuchtung nun gut darstellbar. Als Komplikationen werden Pneumothoraces mit Anlage einer erforderlichen Thoraxsaugdrainage bei $6-15 \%$ der Patienten beschrieben, desweiten traten Blutungen sowie thorakale Schmerzen in bis zu 11 Prozent der Fälle nach der Intervention auf (Watanabe et al. 2006, Kawanaka et al. 2009). Bei versehentlicher Applikation in die Pulmonalvenen, ist die Gefahr eines Hirninfarkts gegeben. Insgesamt stellt dieses Verfahren jedoch eine relativ einfach durchzuführende und preiswerte Markierungsmethode mit guten Erfolgsraten dar (Nomori et al. 2002).

\subsubsection{Präoperative Markierung mit Radionukleotiden}

Die Markierung kleiner Lungenrundherde mit Hilfe von Radionukleotiden findet präoperativ in Lokalanästhesie statt. Eine 22-Gauge-Nadel wird CT-gestützt in die Thoraxwand eingeführt. Nach Sicherung der optimalen Lage der Nadelspitze im Rundherd, bzw. in dessen unmittelbarer Nähe, wird die radioaktive Lösung appliziert. Diese besteht aus einem nichtionischen Kontrastmittel sowie Serum-Humanalbumin, markiert mit Technetium-99m. Die intraoperative Lokalisation erfolgt durch eine Gamma-Kamera. Die von dem Messfühler detektierten Gamma-Strahlen werden in akustische sowie digitale Signale umgewandelt. Das akustische Signal steigt nun proportional zur vorhandenen Radioaktivität in dem jeweiligen Gebiet an. Die digitalen Signale werden in Graphenform auf einem Monitor wiedergegeben. 
Somit kann der Rundherd in dem Gebiet mit der höchsten Radioaktivität identifiziert und reseziert werden. Die Detektionsrate liegt bei diesem Verfahren zwischen 96 und 100 Prozent. Nachteilig ist der relativ hohe technische Aufwand mit dem Gebrauch einer Gamma-Kamera sowie die Strahlenbelastung, welcher der Patient ausgesetzt wird (Chella et al. 2000, Gonfiotti et al. 2007).

\subsubsection{Präoperative Drahtmarkierung}

Die CT-gestützte, perkutane Drahtmarkierung kleiner Lungenrundherde stellt ein weiteres präoperatives Markierungsverfahren dar und ist seit Jahren etabliert und in klinischer Anwendung. Verschiedene Markierungsdrähte werden genutzt, wobei man zwischen Haken(Ciriaco et al. 2004, Chen et al. 2007, Gonfiotti et al. 2007) und Spiraldrähten (Eichfeld et al. 2005, Krüger et al. 2006) unterscheidet. Allen gemeinsam ist die präoperativ möglichst nahe Einbringung des Drahtes an den Rundherd. Intraoperativ kann der Operateur dann anhand des Drahtverlaufs den Rundherd sicher lokalisieren. Komplikationen sind selten. Es können Blutungen im Stichkanal sowie Pneumothoraces auftreten, welche jedoch in den meisten Fällen keine weiterführende Behandlung benötigen. Eine weitere Komplikation stellt die Möglichkeit der Dislokation des Markierungsdrahtes dar, welche bei der Umlagerung des Patienten oder intraoperativ bei der Deflation der Lunge auftreten kann.

\subsubsection{Präoperative Markierung mit dem Lungen-Marker-System der Firma} Somatex ${ }^{\circledR}$

Die perkutane, CT-gestützte Einbringung des speziellen spiralförmigen Markierungsdrahtes für Lungenherde der Firma Somatex ${ }^{\circledR}$ (Medical Technologies GmbH, Teltow, Germany) erfolgt in Lokalanästhesie. Mehrere Komponenten bilden dieses Markersystem: Es besteht aus einer Führungsnadel mit Mandrin sowie einer angeschliffenen Hohlnadel (1,2 mm Durchmesser, 18 Gauge), welche den Markierungsdraht enthält. Dieser nimmt erst nach Entfaltung seine vorgegebene Spiralform an. Nach der Lagerung des Patienten auf dem CTTisch wird zunächst ein Planungs-CT zur Festlegung des optimalen Zugangsweges durchgeführt. Für eine erfolgreiche Markierung ist die klare vorherige Instruktion der 
Patienten, bei jedem Atemkommando immer dieselbe Atemtiefe einzuhalten - vom Planungs-CT bis zur Abschlusskontrolle - wichtig. Vor der Hautdesinfektion erfolgen zunächst die Bestimmung des Einstichwinkels und die Markierung der geeigneten Einstichstelle. Nun wird das Lokalanästhetikum injiziert. Das Einbringen der Führungsnadel wird unter computertomographischer Lagekontrolle durchgeführt. Ist die richtige Position gesichert, kann der Mandrin entfernt und durch die Hohlnadel mit inliegendem Markierungsdraht ersetzt werden. Nach Sicherung der richtigen Nadelposition im bzw. möglichst nah am Rundherd, wird der Draht vorgeschoben und entwickelt seine ursprüngliche Spiralform. Anschließend erfolgt der Nadelrückzug. Ist die korrekte Drahtposition durch einen weiteren Kontroll-Scan bestätigt, kann der Draht transthorakal fixiert werden. Der Patient kann nun sofort mit liegendem Draht in den OP gebracht werden oder geht vorübergehend auf Station zurück. Die Operation findet jedoch immer noch am selben Tag statt.

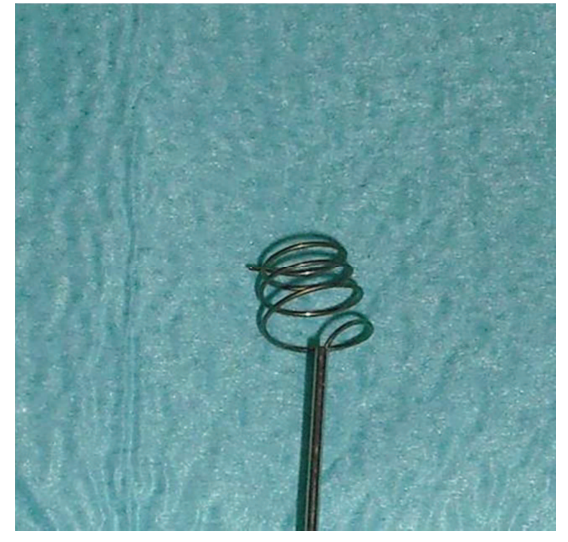

Markierungsdraht der Firma Somatex ${ }^{\circledR}$

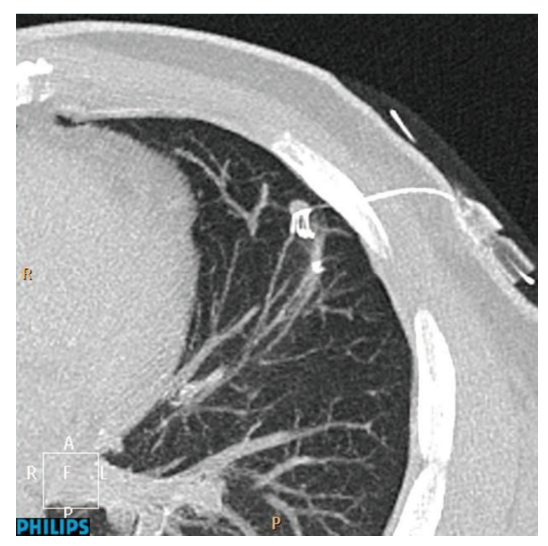

Abschluss-Kontroll-Scan nach Markierung des Rundherds, MIP-Rekonstruktion (Maximumintensitätsprojektion) 


\subsection{Zielsetzung}

Die Zielsetzung dieser Arbeit bestand in der Beurteilung der Wertigkeit der präoperativen

CT-gestützten Drahtmarkierung von kleinen, peripher gelegenen Rundherden bei der Verwendung des Lungen-Marker-Systems der Firma Somatex ${ }^{\circledR}$ vor videothorakoskopischer Resektion.

Wir berichten über unsere Erfahrung mit diesem speziellen System bei 184 Markierungen in dem Zeitraum von Oktober 1999 bis März 2010. 
3. Publikation

DOI 10.1055/s-0031-1299418

Online-Publikation: 2012

(C) Georg Thieme Verlag

\section{CT-gestützte Drahtmarkierung vor videoassistierter thorakoskopischer OP von pulmonalen Rundherden - eine Auswertung von 184 Fällen}

CT-Guided Marking of Pulmonary Nodules with a Special Lung Marking Wire

Before Video-Assisted Thoracoscopic Surgery - Review of 184 Cases

M.-K. Schulze, U. Eichfeld, T. Kahn, P. Stumpp

Klinik und Poliklinik für Diagnostische und Interventionelle Radiologie, Universitätsklinikum Leipzig AöR 


\title{
CT-gestützte Drahtmarkierung vor videoassistierter thorakoskopischer OP von pulmonalen Rundherden - eine Auswertung von 184 Fällen
}

\author{
CT-Guided Marking of Pulmonary Nodules with a Special Lung Marking Wire \\ Before Video-Assisted Thoracoscopic Surgery - Review of 184 Cases
}

Autoren

Institut
M.-K. Schulze, U. Eichfeld, T. Kahn, P. Stumpp

Klinik und Poliklinik für Diagnostische und Interventionelle Radiologie, Universitätsklinikum Leipzig AöR

\author{
Key words \\ - thorax \\ - CT spiral \\ thoracoscopy \\ - interventional procedures
}

eingereicht 19.8.2011 akzeptiert 16.2.2012

Bibliografie

DOI http://dx.doi.org/ 10.1055/s-0031-1299418 Online-Publikation: 2012

Fortschr Röntgenstr @ Georg Thieme Verlag KG Stuttgart . New York · ISSN 1438-9029

\section{Korrespondenzadresse}

Miss Marie-Kristin Schulze Klinik und Poliklinik für Diagnostische und Interventionelle Radiologie, Universitätsklinikum Leipzig AöR

Liebigstr. 20

04103 Leipzig

Tel.: ++49/341/9717400

Fax: $++49 / 341 / 9717409$

marie-kristin-schulze@web.de

\section{Zusammenfassung \\ $\nabla$}

Ziel: Minimalinvasive Techniken, wie die videoassistierte Thoraxchirurgie (VATS), sind heutzutage zur Resektion vor allem peripher gelegener Rundherde Methode der Wahl. Um eine schnelle und sichere intraoperative Lokalisation zu gewährleisten, haben sich diverse Markierungstechniken etabliert. Wir berichten über die Erfahrungen bei 184 Markierungen mittels eines speziellen Lungenmarkierungsdrahts, der CT-gestützt präoperativ an den Herd gebracht wird.

Material und Methoden: Bei 184 Patienten (97 m, 87w, mittleres Alter: 58,1 $\pm 13,7$ Jahre) wurde unmittelbar vor der VATS eine CT-gesteuerte Markierung des Rundherds mithilfe eines Spiraldrahts vorgenommen. Evaluiert wurden anschließend die Erfolgsrate, die Sicherheit der Intervention, die Notwendigkeit eines Umstiegs zur Thorakotomie sowie die Histologie der Patienten.

Ergebnisse: Der Markierungsdraht konnte in 181 Fällen $(98,4 \%)$ erfolgreich platziert werden. Eine Markierung musste aufgrund eines nicht beherrschbaren Pneumothorax abgebrochen werden. Geringgradige, methodisch nicht vermeidbare Komplikationen wie Mantelpneumothoraces $(53,3 \%)$ oder perifokale Blutungen $(30,4 \%)$ bedurften keiner Therapie. Die makroskopisch vollständige Entfernung der markierten Herde gelang bei 98,4\% der Patienten. Aufgrund intraoperativer Komplikationen (Adhäsionen, Blutungen, Drahtdislokationen) war in 29 Fällen (15,9\%) die Konversion zur Thorakotomie erforderlich. Die Histologie ergab in 96 Fällen (52,5\%) einen benignen Befund, in 87 Fällen (47,5\%) einen malignen, wobei insgesamt lediglich 21 Herde $(11,5 \%)$ einem primären Lungenkarzinom entsprachen.

Schlussfolgerung: Die Kombination aus CT-gesteuerter Herdfixierung mittels Spiraldraht und die anschließende thorakoskopische Entfernung ist eine effiziente und sichere Methode zur Diag-

\section{Abstract}

V

Purpose: Minimally invasive techniques like video-assisted thoracoscopic surgery (VATS) are currently the method of choice for the resection of small pulmonary nodules, when they are located in the periphery of the lungs. To guarantee quick and safe intraoperative identification of the nodule, preoperative marking is necessary and sensible. We report about our experiences in 184 markings with a special lung marking wire, which is placed in or around the pulmonary nodule using CT guidance.

Materials and Methods: In 184 patients $(97 \mathrm{~m}$, 87 , mean age: $58.1 \pm 13.7$ years) with pulmonary nodules, scheduled for resection with VATS, a special lung marking wire was placed preoperatively under CT guidance. We evaluated the technical success, safety, necessity of conversion to thoracotomy and histology in all patients.

Results: The marking wire could be positioned successfully in 181 cases (98.4\%). There was one major complication (uncontrollable pneumothorax). Minor adverse events like small pneumothorax $(53.3 \%)$ or a perifocal bleeding $(30.4 \%)$ did not necessitate treatment. Complete resection of the marked nodule was successful in $98.4 \%$ of the patients. Conversion to thoracotomy was necessary in 29 patients (15.9\%) due to bleeding, adhesions, malignancy or wire dislocation. Histology revealed a benign nodule in 96 cases (54.4\%) and a malignant lesion in 78 cases $(45.6 \%)$, of which only 21 nodules (11.5\%) turned out to present a primary pulmonary carcinoma.

Conclusion: CT-guided marking of pulmonary nodules using a special marking wire followed by thoracoscopic resection is an efficient and safe method for diagnosing suspicious nodules in the periphery of the lung. 
nostik von unklaren intrapulmonalen Rundherden, insbesondere für periphere, subpleural gelegene Herde.

\section{Einleitung}

\section{$\nabla$}

Als pulmonaler Rundherd ( $\bullet$ Abb. 1a) werden mehr oder weniger runde Verschattungen bezeichnet, welche vollständig von Lunge umgeben sind und deren Durchmesser weniger als $3 \mathrm{~cm}$ beträgt. In $90 \%$ der Fälle handelt es sich um Zufallsbefunde, pro 500 Röntgenaufnahmen wird ca. ein Rundherd entdeckt [1]. Im Durchschnitt sind $50 \%$ aller Rundherde maligne, wobei die Streubreite in den Studien von $10-70 \%$ sehr variabel ist, je nachdem, welches Patientenkollektiv für die jeweilige Studie gewählt wurde. Ein radiologisch erfasster, neu entdeckter Lungenrundherd ist somit immer als malignitätsverdächtig anzusehen und macht eine weitere Abklärung nötig. Die Wahrscheinlichkeit der Malignität steigt mit zunehmender Größe des Herdes, Alter des Patienten, bekanntem Nikotinabusus oder einer Karzinomerkrankung in der Vorgeschichte. Kalzifizierungen, Fetteinlagerungen oder eine Größenkonstanz sprechen dagegen eher für ein benignes Geschehen. Die Fleischner Society [2] empfiehlt derzeit folgendes Vorgehen zur radiologischen Abklärung zufällig entdeckter Rundherde: Da 99\% aller Herde $\leq 4 \mathrm{~mm}$ eine benigne Histologie aufweisen, wird nur in Fällen einer suspekten Morphologie bzw. bei Hochrisikopatienten ein Follow-up-CT in 12 Monaten empfohlen. Nicht kalzifizierte Rundherde $\geq 8 \mathrm{~mm}$ dagegen sollten einer unverzüglichen Abklärung zugeführt werden. In Abhängigkeit der individuellen Umstände sollten entweder Follow-up-Scans oder eine umgehende Histologiegewinnung empfohlen werden. Verallgemeinernd lässt sich sagen, dass in jedem einzelnen Fall individuell entschieden werden muss, inwieweit ein Rundherd als malignitätsverdächtig einzustufen ist. Daran gemessen kann zunächst ein konservatives Vorgehen vor einem sofortigen invasiven Verfahren in Betracht gezogen werden.

Trotz der aktuell hochqualitativen bildgebenden Verfahren lässt sich die Dignität einer Läsion mit einem nicht invasiven Verfahren nicht zweifelsfrei klären. Eine histologische Sicherung ist in der Mehrzahl der Fälle unumgänglich, wobei verschiedene Verfahren, je nach Lage und Größe des Herdes, zur Verfügung stehen.
Größere zentral gelegene Herde können meist mit der transbronchialen Biopsie im Rahmen einer Bronchoskopie gut erreicht werden. Die transthorakale CT-gestützte Punktion eignet sich zur Sicherung peripherer Rundherde ab einer Größe von ca. $1 \mathrm{~cm}$. Kleinere Herde lassen sich mit dieser Methode jedoch schwer erreichen und erlauben nur eine eingeschränkte Aussagekraft der Punktion. In diesen Fällen kommt der thorakoskopischen Resektion eine entscheidende Rolle zu. Sie ist Methode der Wahl, um kleine, peripher gelegene Rundherde histologisch repräsentativ zu sichern. Dank der Verbesserung und Weiterentwicklung medizinischer Techniken stellt die videoassistierte thorakoskopische Chirurgie (VATS) heutzutage eine Alternative zur konventionellen Thorakotomie dar. Pulmonale Rundherde können zu diagnostischen wie auch therapeutischen Zwecken minimalinvasiv entfernt werden [3, 4]. Neben der kürzeren Krankenhausaufenthaltsdauer und weniger postoperativen Schmerzen stellen geringere Morbidität und Mortalität Vorteile für die Patienten dar [5]. Die Hauptlimitation in der Anwendung der VATS bestand lange darin, dass die Herde entweder zu klein waren oder zu weit von der pleuralen Oberfläche entfernt lagen, um rein thorakoskopisch, also ohne digitale Palpation, lokalisiert zu werden. Auch topografische Beziehungen der Zielherde zu anatomischen Landmarken auf der Hautoberfläche, oder intrathorakal, gehen durch den Lungenkollaps und den iatrogen erzeugten Pneumothorax während der Prozedur verloren. Aufgrund dessen wurden vielfältige Methoden zur präoperativen Markierung der Läsionen entwickelt und stellten sich als vorteilhaft heraus. Ciriaco et al. [6] und Chen und Kollegen [7] verwendeten Hakendrähte, Partrick und Kollegen [8] sowie Willekes et al. [9] nutzten Methylenblau zur Anfärbung. Radionukleotide wurden von Boni und Kollegen [10] verwendet, Kawanaka et al. [11] nutzten Lipiodol zur präoperativen Markierung. All diese verschiedenen Verfahren haben ihre jeweiligen Vor- und Nachteile. Als ungünstig erwies sich bei der Verwendung von gefärbten Substanzen das Auslaufen in umliegendes Gewebe und somit die Maskierung des Herdes, Radionukleotide haben eine höhere Strahlenbelas-
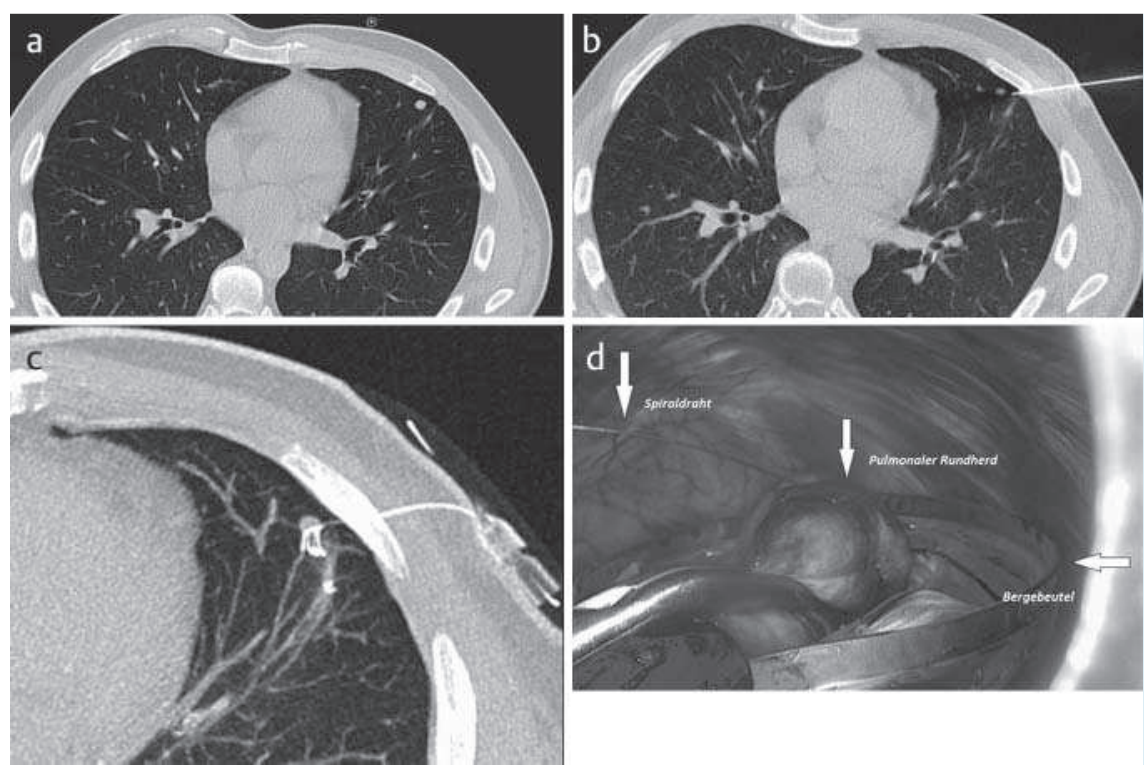

Abb. 1 a Pulmonaler Rundherd im linken Oberlappen. b CT-Kontroll-Scan nach der Punktion mit der Führungsnadel. c Abschluss-Kontroll-Scan nach Markierung des Rundherds (MIP-Rekonstruktion). d Bergung des Herdes mit Spiraldraht nach atypischer Resektion im Bergebeutel: 


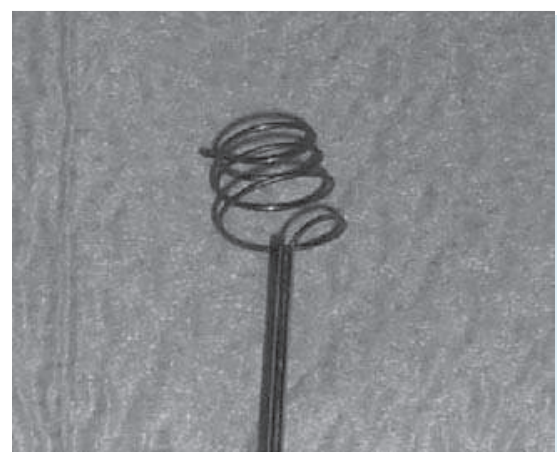

Abb. 2 Markierungsspirale (Somatex, Berlin, Deutschland).

tung für den Patienten zur Folge. Lipiodol, ein nicht wasserlösliches Röntgenkontrastmittel, birgt die Gefahr eines Hirninfarkts bei versehentlicher Applikation in die Pulmonalvenen.

An unserer Einrichtung wird seit ca. 10 Jahren die präoperative Herdmarkierung mit einem speziell dafür entwickelten Draht (Somatex Medical Technologies GmbH, Berlin, Deutschland) ( $\bullet$ Abb. 2) durchgeführt. Wir berichten hier über unsere Erfahrungen mit 184 Patienten, die sich der Kombination aus CTgesteuerter, perkutaner Herdmarkierung und anschließender videoassistierter, rein thorakoskopischer OP unterzogen haben. Bei allen Patienten wurden vorher mittels CT-Untersuchungen malignitätssuspekte oder zumindest abklärungsbedürftige Rundherde nachgewiesen, welche entweder bei Staging- oder Follow-up-Untersuchungen bzw. als Zufallsbefunde im Rahmen anderer diagnostischer Maßnahmen auffielen. Nach interdisziplinärer Absprache innerhalb von Tumorboards oder chirurgisch/radiologischen Besprechungen wurde anschließend (angelehnt an die Empfehlungen der Fleischner Society [2]) die Indikation zur Drahtmarkierung mit darauffolgender VATS-Resektion gestellt. Diese 184 Fälle entsprechen einem zufälligen Patientenkollektiv, welches retrospektiv erfasst und ausgewertet wurde.

\section{Patienten und Methode \\ $\nabla$}

Die Auswahl der Patienten erfolgte mit einer elektronischen Suche im radiologischen Informationssystem anhand des Suchbegriffs „Drahtmarkierung“. Dabei ergab der erste Suchlauf eine Trefferzahl von 497. Nach eingehender Analyse der Einzelfälle konnten 184 Patienten identifiziert werden, welche sich in dem Zeitraum von Oktober 1999 bis März 2010 einer Drahtmarkierung mit anschließender VATS unterzogen haben. Bei den verbliebenen 313 Patienten wurden zum Teil andere Drahtmarkierungen (z.B. Markierungen der Mamma) vorgenommen oder eine Drahtmarkierung wurde lediglich im Text empfohlen. Diese Patienten wurden daher für die weitere Auswertung nicht berücksichtigt.

Es folgte die Datenanalyse mithilfe des Picture Archiving and Communication System (PACS). Die Bilddaten wurden auf einer MagicView 1000-Workstation ${ }^{\circledR}$ (Siemens Health Services, Erlangen, Deutschland) bezüglich folgender Punkte evaluiert:

- Lokalisation/Größe der Herde

- Abstand zur pleuralen Oberfläche

- Charakterisierung der Herde (Form, Begrenzung)

- Markierungsdauer, Anzahl nötiger Kontroll-Scans

- Anzahl eventuell nötiger Lagekorrekturen
Tab. 1 Detektion der Rundherde.

\begin{tabular}{|llll|} 
& Zufallsbefund & $\begin{array}{l}\text { Nachuntersuchung } \\
\text { bei maligner } \\
\text { Grunderkrankung }\end{array}$ & $\begin{array}{l}\text { Staging- } \\
\text { untersuchung }\end{array}$ \\
\hline Anzahl & 62 & 94 & 28 \\
\hline $\begin{array}{l}\text { Anteil } \\
\text { (in \%) }\end{array}$ & 33,7 & 51,1 & 15,2 \\
\hline
\end{tabular}

Tab. 2 Lokalisation der Rundherde.

\begin{tabular}{|c|c|c|}
\hline & rechts & links \\
\hline Anzahl gesamt & 111 & 73 \\
\hline Oberlappen & 41 & 30 \\
\hline Mittellappen & 20 & n. a. \\
\hline Unterlappen & 50 & 43 \\
\hline
\end{tabular}

- Abstand zwischen Draht und Herd sowie Drahtspitze und Pleura

- Komplikationen (Pneumothorax, Blutungen, Drahtdislokation) Die anschließende Einsicht in sämtliche OP-Berichte ermöglichte es, die Dauer zwischen der Markierung und dem Eingriff zu bestimmen und Komplikationen intraoperativ zu erfassen. Dabei wurden folgende Punkte analysiert:

- Dauer zwischen Markierung und VATS

> OP-Dauer

- Komplikationen

- Vollständige Entfernung des Herdes?

- Konversion zur Thorakotomie nötig? Wenn ja, welche Ursache? - Histologie

62 (33,7\%) der Läsionen waren Zufallsbefunde, 94 (51,1\%) wurden im Rahmen von Nachuntersuchungen bei bekannter maligner Grunderkrankung detektiert und 28 (15,2\%) der Herde wurden während Staginguntersuchungen diagnostiziert ( $\bullet$ Tab. 1).

Das Durchschnittsalter lag bei 58,1 Jahren, wobei der jüngste Patient ein Alter von 6 Jahren hatte, der älteste war 79.

Die Lokalisation stellte sich folgendermaßen dar: 111 Herde (60,3\%) befanden sich auf der rechten Seite, davon 41 im Ober-, 20 im Mittel- und 50 im Unterlappen. Linksseitig fanden sich 30 im Ober- sowie 43 im Unterlappen ( $\bullet$ Tab. 2).

Zur Ermittlung der Größe der Herde wurde die maximale Ausdehnung in axialer Schichtung ermittelt. Diese betrug durchschnittlich 7,0×9,7 mm, die Tiefe der Läsionen lag im Mittel bei $9,8 \pm 9,1 \mathrm{~mm}$.

\section{CT-gesteuerte Markierung}

Die präoperative Einbringung des Markierungsdrahts der Firma Somatex $^{\circledR}$ (Medical Technologies GmbH, Teltow, Germany) erfolgt in Lokalanästhesie. Dieses spiralförmige Markierungssystem besteht aus mehreren Komponenten: einer Führungsnadel mit Mandrin sowie einer weiteren Hohlnadel und dem dort einliegenden Spiraldraht. Nach Lagerung des Patienten auf dem CTTisch wird das Planungs-CT durchgeführt. Für eine erfolgreiche Markierung ist die klare vorherige Instruktion der Patienten, bei jedem Atemkommando immer dieselbe Atemtiefe einzuhalten, vom Planungs-CT bis zur Abschlusskontrolle, wichtig. An die Definition der Schichtebene (z-Achse) schließt sich die Planung des Zugangswegs ( $\mathrm{x}-\mathrm{y}$-Achse) an. Die Hautdesinfektion erfolgt nach Markierung der Einstichstelle und Bestimmung des geeigneten Einstichwinkels. Anschließend wird das Lokalanästhetikum in 
die Thoraxwand injiziert. Unter CT-gestützter Lagekontrolle erfolgt nun das Einbringen der Führungsnadel. Befindet sich diese in richtiger Position, kann der Mandrin entfernt und durch die Hohlnadel mit inliegendem Markierungsdraht ersetzt werden. Ist die korrekte Nadelposition möglichst nah am bzw. im Rundherd gesichert, wird der Draht vorgeschoben, welcher nach Entfaltung seine ursprüngliche Form annimmt ( $\bullet$ Abb.1b, c). Nach Rückzug der Nadeln und korrekter Lagekontrolle wird der Draht transthorakal fixiert und das externe Ende mit sterilen Kompressen bedeckt.

Die zwischenzeitlichen Kontrollen werden mithilfe eines In-roomBediensystems angefertigt, wobei jeweils 4 Einzelschnitte mit einer Schichtdicke von je 3,75 mm dargestellt werden. Die Abschlusskontrolle ( $\bullet$ Abb. 1c) hingegen ist eine Spiral-CT, aus der MIPs rekonstruiert werden können (axial, sagittal, koronar), welche zur besseren Visualisierung des Drahtverlaufs für den Operateur dienen.

Der Patient kann sofort mit liegendem Draht in den OP gebracht werden oder geht vorübergehend auf Station zurück. Die Operation erfolgt jedoch immer noch am selben Tag.

\section{VATS (video-assisted thoracoscopic surgery)}

Die Indikationen zur VATS sind breit gefächert. Neben beispielsweise Pleurodesen, Probeexzisionen an der Pleura, Versorgung rezidivierender Pneumothoraces, Hämatothoraces sowie Pleuraempyemen stellen unklare Lungenrundherde eine wichtige Indikation für dieses minimalinvasive Verfahren dar.

Die Resektion der markierten Herde erfolgt über lediglich 3, unter Umständen 4, kleine Inzisionen (von ca. $1,5 \mathrm{~cm}$ ) im Bereich der Thoraxwand. Dabei erfolgt bei dem durch einen Doppellumentubus intubierten Patienten, nach Ausklemmen der betroffenen Lungenseite von der Beatmung, zunächst die Thorakoskopie. Nach Identifizierung des gesuchten Herdes anhand des Drahtverlaufs wird dieser durch leichten Zug am externen Anteil des Drahtes in Richtung der inneren Thoraxwand verlagert. Zusätzlich wird der markierte Herd nun durch eine endoskopische Fasszange fixiert. Über die dritte Inzision erfolgen anschließend unter CT-Bildvergleich die Resektion mittels Endo-linear-Cutter ${ }^{\circledR}$ (Ethicon, Norderstedt, Deutschland) sowie die Bergung des Rundherds. Mit dem intrathorakal gelegenen Drahtabschnitt wird der markierte Herd atypisch reseziert, in einen Bergebeutel verbracht und über eine Thoraxinzision exstirpiert ( $\bullet$ Abb. 1 d). Zuvor wird der Draht auf Hautniveau durchtrennt. Das entnommene Gewebe wird zum Schnellschnitt und zur Histologie dem Pathologen übergeben. Nach Kontrolle auf Luftdichtigkeit und Ausschluss von Blutungen erfolgt nach Einlage einer Thoraxdrainage der Wundverschluss.

\section{Ergebnisse \\ $\nabla$}

Eine erfolgreiche Platzierung des Drahtes in bzw. am Rundherd konnte in 98,4\% der Fälle erreicht werden. Bei 2 Patienten kam es während der Markierung zu einer Drahtdislokation. In einem Fall musste die Markierung aufgrund eines nicht kontrollierbaren Pneumothorax abgebrochen werden. Bei den verbliebenen 181 Patienten konnte der Herd problemlos markiert und fixiert werden. Die Markierungsdauer beanspruchte dabei 11 - $79 \mathrm{~min}$ mit einer mittleren Markierungszeit von $31 \pm 10$ min. Bei 108 der Patienten verlief der Draht durch die Läsion hindurch oder lag zentral im Herd. In den anderen 75 Fällen konnte er im umliegenden Gewebe platziert werden, wobei der mittlere Herd-Draht-Ab-

\begin{tabular}{|l|lll|}
\hline \multicolumn{1}{|c|}{ Tab.3 } & Komplikationen. & & \\
& Pneumothorax & Einblutung & Dislokation \\
\hline Anzahl & 98 & 56 & 6 \\
\hline Anteil (in \%) & 53,3 & 30,4 & 3,3 \\
\hline
\end{tabular}

Tab.4 Zusammenhang zwischen regulärer/irregulärer Begrenzung und primärem Lungen-CA/Metastase.

\begin{tabular}{|llll|}
\hline & & regulär & irregulär \\
\hline Primum & Anzahl & 8 & 13 \\
\hline & Anteil (in \%) & 38,1 & 61,9 \\
\hline Metastase & Anzahl & 50 & 16 \\
\hline & Anteil (in \%) & 75,8 & 24,2 \\
\hline
\end{tabular}

stand 2,47 $\pm 4,57 \mathrm{~mm}$ betrug, im extremsten Fall lag das spiralförmige Drahtende $3 \mathrm{~cm}$ zentral des Herdes. Dieser Umstand wurde dem Chirurgen mitgeteilt und intraoperativ berücksichtigt, sodass auch hier die thorakoskopische Resektion nach Rückzug durch Rückdrehung der Spirale und unter Sicht problemlos durchgeführt werden konnte. Im Durchschnitt wurden 7,3 \pm 7,0 Kontroll-Scans benötigt, um die exakte Drahtlage zu verifizieren. Nadelrepositionen wurden im Mittel nur 1,3 $\pm 2,2$-mal vollzogen. Als Minor-Komplikationen fanden sich Einblutungen im Stichkanal und Pneumothoraces (mit einer Ausdehnung von 2-68 mm, Durchschnittswert: 10,6 mm). Bei 6 Patienten kam es während der Markierung in der Zeit zwischen Markierung und OP bzw. während der OP-Lagerung zu einer Drahtdislokation ( $\bullet$ Tab.3). In 4 dieser Fälle konnte dennoch eine VATS durchgeführt werden, da die Zielregion anhand der verursachten lokalen Blutung erkannt werden konnte. Zweimal war aufgrund dessen die Konversion zur Thorakotomie erforderlich. Insgesamt entschloss man sich in 29 Fällen (15,9\%) auf eine Thorakotomie umzusteigen, Gründe dafür waren neben den Drahtdislokationen starke Adhäsionen, Blutungen sowie eine intraoperativ festgestellte Malignität. In diesen Fällen ist die Entscheidung zwischen einem sofortigen Umstieg zur Thorakotomie und einem zweizeitigen Eingriff immer individuell zu stellen und von der speziellen Situation abhängig. Voraussetzungen für eine sofortige Thorakotomie sind ein im Schnellschnitt sicher bestätigtes nicht kleinzelliges Bronchialkarzinom und dass präoperativ bereits alle Staging- und Lungenfunktionsuntersuchungen stattgefunden haben. Somit kann häufig eine Zweitoperation vermieden werden. Des Weiteren können bei einer Metastasensicherung das offene Austasten der Lunge sowie eine minimalinvasive R1-Resektion maligner Befunde einen Umstieg erfordern.

Die mittlere Krankenhausaufenthaltsdauer nach durchgeführter Resektion betrug 8,4 $\pm 5,1$ Tage mit einer Spannweite von 2-47 Tagen, wobei bei diesem Patienten die lange Liegedauer durch sein malignes Grundleiden mit sich z.T. unmittelbar anschließenden Karzinomoperationen und nötiger Metastasenchirurgie (Nierenzellkarzinom) bedingt war.

Die histologische Differenzierung ergab bei 96 Patienten (52,5\%) benigne Ergebnisse, in 87 Fällen (47,5\%) zeigten sich maligne Erkrankungen. Die primären Lungenkarzinome zeigten in $61,9 \%$ eine irreguläre Begrenzung, wohingegen Metastasen nur in 24,2\% eine solche aufwiesen und sich in der Mehrzahl der Fälle $(75,8 \%)$ mit regulärer, glatter Umrandung darstellten (๑ Tab.4). 


\begin{tabular}{|llll|}
\hline benigne: & $\mathbf{9 6}(\mathbf{5 2 , 4 6} \mathbf{\% )}$ & maligne: & $\mathbf{8 7}$ (47,54\%) \\
\hline Anthrakose/Fibrose/Lymphknoten: & 36 & primäres Lungen-CA: & 21 \\
\hline Entzündungsreaktion: & 15 & Adenokarzinom: & 8 \\
\hline Hamartom/Hamartochomdrom: & 11 & Plattenepithelkarzinom: & 7 \\
\hline Granulom: & 9 & kleinzelliges Karzinom: & 2 \\
\hline Emphysem: & 5 & neuroendokrines CA: & 2 \\
\hline Nekrose: & 4 & großzelliges Karzinom: & 1 \\
\hline Sarkoidose: & 4 & Hämangioendotheliom: & 1 \\
\hline normales Lungengewebe: & 3 & & \\
\hline Aspergillom: & 2 & Metastase: & $\mathbf{6 6}$ \\
\hline Amyloidose: & 2 & Adenokarzinom: & 32 \\
\hline Abszess: & 2 & Plattenepithelkarzinom: & 8 \\
\hline Lymphangioleiomyomatose: & 1 & Sarkom: & 7 \\
\hline Narbengewebe: & 1 & neuroendokrines CA: & 4 \\
\hline Rheumatoid. Arthritis: & 1 & kleinzelliges Nieren-CA: & 4 \\
\hline & & Lymphom: & 3 \\
\hline
\end{tabular}

Tab. 5 Histopathologische Differenzierung der resezierten Rundherde.
Bei den benignen Herden handelte es sich meist um anthrakotische oder fibrotische Lymphknoten $(n=36,37,5 \%)$ sowie entzündliche Geschehen ( $\mathrm{n}=15,15,6 \%)$. Weiterhin fanden sich u.a. Hamartome, Hamartochondrome, Aspergillome, Abszesse, Nekrosen und Amyloidose-Herde.

Bei den malignen Läsionen handelte es sich um 66 Metastasen und 21 primäre Lungenkarzinome, worunter sich 8 Adeno-, 7 Plattenepithel-, 2 neuroendokrine, 2 kleinzellige sowie ein großzelliges Karzinom befanden. Des Weiteren ergab die histologische Untersuchung ein niedrig malignes Hämangioendotheliom. Von den 66 Patienten mit diagnostizierten Metastasen litten 57 bereits an einer malignen Grunderkrankung, in 9 Fällen wurden sie während Staging-Untersuchungen im Rahmen kürzlich diagnostizierter Malignome erkannt. Den Hauptanteil bildeten mit 36,8\% Streuherde von Adenokarzinomen, gefolgt von Plattenepithelkarzinomen und Sarkomen. Weiterhin zeigten sich Metastasen neuroendokriner Karzinome, kleinzelliger Nierenkarzinome und Lymphommanifestationen in der Lunge ( $\bullet$ Tab.5).

\section{Diskussion}

$\nabla$

Die videoassistierte Thorakoskopie (VATS) stellt eine wertvolle Ergänzung zu den konventionellen Operationsverfahren in der Thoraxchirurgie dar. Sie steht dank modernster Videotechniken für eine Vielzahl von diagnostischen und therapeutischen Verfahren im Bereich des Lungenparenchyms und der Pleura zur Verfügung. Zu den Indikationsfeldern zählen neben diagnostischen Probeexzisionen unklarer Herde u. a. die Behandlung therapiebedürftiger Hämatothoraces sowie Lymphknotendissektionen. Resektionen umfassen neben peripheren Tumoren an der Lunge auch atypische Segment- bis hin zu minimalinvasiven Lappenresektionen [5, 12 - 15].

Unstrittige Vorteile dieser minimalinvasiven Operationstechnik sind eine geringere Morbidität und Mortalität, geringere postoperative Schmerzen [16] sowie eine kürzere Krankenhausaufenthaltsdauer [4, 17]. Auch in unserer Patientengruppe hatten Patienten nach VATS im Vergleich zu thorakotomierten Patienten eine kürzere mittlere postoperative Krankenhausaufenthaltsdauer (7,91 Tage). Nach der Resektion eines benignen Rundherds ohne weitere Komplikationen betrug diese durchschnittlich 6,80 Tage. Diese dennoch relativ lange Krankenhausaufenthaltsdauer lässt sich am ehesten durch Komorbiditäten der Patienten mit entsprechend verlängertem Behandlungsbedarf erklären. Patienten, bei welchen ein Umstieg auf eine Thorakotomie aufgrund von Adhäsionen, malignen Erkrankungen oder Blutungen unumgänglich war, mussten postoperativ im Mittel 10,83 Tage im Krankenhaus verbleiben.

Der kosmetische Aspekt mit deutlich kleineren Narben stellt einen weiteren Vorteil der VATS gegenüber der offenen Thorakotomie dar, der für viele Patienten ebenso bedeutsam ist.

Eine Einschränkung besteht in der Bedingung der relativ peripheren Lage des zu resezierenden Herdes. Eine sichere videoassistierte Resektion eines suspekten Befunds ist somit nur für Herde möglich, welche sich in relativer Nähe zur Pleura befinden. Um eine komplikationsarme und schnelle intraoperative Lokalisation vor allem kleiner, peripherer, subpleural gelegener Herde zu gewährleisten, welche bei einer endoskopischen OP weder tast- noch sichtbar sind, haben sich präoperative Markierungstechniken fest etabliert. Suzuki et al. [18] berichten über eine Nichtdetektierbarkeit der Herde von mehr als $50 \%$, sollten diese einen Abstand zur Pleura $>5 \mathrm{~mm}$ bzw. eine Größe $\leq 10 \mathrm{~mm}$ aufweisen. Ab einem Herd-Pleura-Abstand $>10 \mathrm{~mm}$ erachten sie eine präoperative Markierung als absolut indiziert. Ähnliche Ergebnisse fanden Tamura und Kollegen [19] in ihrer Untersuchung: Betrug die Distanz zur pleuralen Oberfläche $>10 \mathrm{~mm}$ und wies der Herd eine Größe $<15 \mathrm{~mm}$ auf, so war der Rundherd thorakoskopisch nicht zu identifizieren.

In unserer Eichrichtung werden kleine drahtmarkierte Herde bis zu einem Abstand von ca. $3 \mathrm{~cm}$ zur viszeralen Pleura mittels VATS reseziert. Eine thorakoskopisch gut erreichbare Lage der Läsion erlaubt in Ausnahmefällen auch die Resektion tiefer gelegener Herde, die Indikation zur Drahtmarkierung stellen dabei immer die Chirurgen. Die tiefste Läsion lag bei uns $47 \mathrm{~mm}$ subpleural. Wir möchten an dieser Stelle noch einmal betonen, dass in unserem Haus sämtliche VATS-Resektionen ohne digitale Austastung vorgenommen werden, d. h. es werden maximal $1,5 \mathrm{~cm}$ lange Inzisionen genutzt, ohne jegliche Schnitterweiterung, welche das Einführen einzelner Finger zur Palpation ermöglichen würde. Die spezifischen Markierungsdrähte unterscheidet man in Anker- bzw. Hakendrähte und Spiraldrähte [6, 7, 20, 21]. Vorteile des von uns verwendeten Spiraldrahts sind folgende: Flexible, dennoch äußerst stabile Materialeigenschaften, welche eine bessere Fixierung durch die spezielle Spiralform im weichen Lungengewebe ermöglichen, eine leichte Handhabung des Markersystems sowie ein biegsames extrathorakales Ende, welches 
sowohl eine äußere Fixierung als auch eine intraoperativ dosierte Zugkraft-Anwendung zulässt. Die Korrigierbarkeit des Drahtsystems mittels der koaxialen Ladekanülentechnik sowie das akzeptable Punktionsrisiko bei der Nutzung einer lediglich 18 Gauge dicken Nadel stellen einen weiteren Benefit dieser Methode dar [20 - 22].

Auch andere Arten der präoperativen Markierung wurden beschrieben, wie z. B. Radionuklide, Lipiodol oder Methylenblau [911]. Der Nachteil gefärbter Substanzen gegenüber der Verwendung von Drähten liegt im Auslaufen der Farbflüssigkeit in das umliegende Gewebe, sodass keine exakte Lokalisierung des Rundherds mehr möglich ist. Darüber hinaus ist die Färbung mit Methylenblau eine relativ kurzlebige Methode, da die Farbe nur für einen kurzen Zeitraum sichtbar bleibt und die VATS innerhalb von ca. $3 \mathrm{~h}$ erfolgen muss [23]. Shah et al. beschreiben die Entwicklung eines Schockzustands bei einem Patienten kurz nach der Injektion von Methylenblau [24]. Die erhöhte Strahlenbelastung des Patienten bei der Nutzung von Radionukliden sowie der intraoperativ notwendige Gebrauch einer Gamma-Kamera zur Lokalisation stellen Nachteile dieser Methode dar. Auch erwies sich die Verwendung von Markierungsdrähten, welche ursprünglich für Brustgewebe entwickelt wurden, als unvorteilhaft, da diese in dem weniger dichten Lungengewebe zu Dislokationen neigen und starke postoperative Schmerzen verursachen [22, 24].

Wie bereits von Kloeppel et al. [20] erstmalig bei 5 Patienten beschrieben, wurden in unserer Untersuchung bei 184 Patienten spezielle spiralförmige Lungenmarkierungsdrähte verwendet und zeigten sehr gute Ergebnisse. Die Drähte wurden präoperativ CTgestützt im bzw. möglichst nah am Rundherd eingebracht. Vorteile dieser bildgebungsgestützten Eingriffe stellen dabei die Möglichkeit der genauen und schnellen Markierung der Einstichstelle sowie wiederholbare Lagekontrollen während der Intervention dar. Diese Eigenschaften sind besonders für die Markierung sehr kleiner Lungenherde wertvoll. Darüber hinaus benötigt die Markierung nur wenig Zeit. Die mittlere Interventionsdauer lag in unserer Klinik bei 30,9 min. Diese Dauer wurde in unserer Arbeit retrospektiv ermittelt und definiert als Zeit zwischen dem PlanungsCT-Bild und der Abschlusskontrolle. Die eigentliche Drahtmarkierung mit Platzierung der Führungsnadel und dem Vorschub des Markierungsdrahts beansprucht in der Regel nur wenige Minuten. Chen et al. [7] berichten über eine durchschnittlich beanspruchte Interventionsdauer von 30,4 min bei der Verwendung von Hakendrähten, Powell und Kollegen [25] nutzten Platin-Microcoils zur Markierung und benötigten dafür im Mittel $42 \mathrm{~min}$.

In unserer Untersuchung konnten 98,4\% der Markierungen erfolgreich und ohne größere Komplikationen durchgeführt werden. Kleinere Komplikationen wie Mantelpneumothoraces, welche bei einer Punktion kaum zu vermeiden sind, oder Blutungen entlang des Stichkanals kamen vor, mussten jedoch nicht akut behandelt werden ( $\bullet$ Tab.3). Frühere Veröffentlichungen spiegeln ähnliche Komplikationsraten wider [26, 27]. Yoshida et al. berichten beispielsweise über Pneumothoraxraten von 49,1\%, kleinere intrapulmonale Blutungen traten in $29,8 \%$ der Fälle auf [28].

Lediglich bei einem Patienten musste die Markierung aufgrund eines nicht beherrschbaren Pneumothorax (mit anschließender Anlage einer Thoraxdrainage) abgebrochen werden. Bei 2 der Patienten kam es während der Markierung zu einer Dislokation des Drahtes. Insgesamt fanden 6 Drahtdislokationen (3,3\%) bis zum Beginn der VATS statt. Bei der Verwendung von Hakendrähten, beschrieben von Chen et al. [7], kam es in 6,9\% der Fälle zu einer Dislokation, Gonfiotti et al. berichten in ihrer Untersuchung über eine Dislokationsrate der Hakendrähte von 16\% [29]. Frühere Studien schildern ebenfalls höhere Dislokationsraten von 6 $20 \%$ bei dem Gebrauch von Mamma-Markierungsdrähten [24, 26, 27].

Die CT-gestützte perkutane Drahtmarkierung von Lungenherden vor thorakoskopischer Resektion ist eine schnelle und komplikationsarme Markierungsvariante und findet zunehmend Anwendung. Dennoch bestehen auch bei diesem Verfahren gewisse Einschränkungen und Risiken. Zu erwarten und - wenn nötig - gut therapierbar ist die Entstehung eines Pneumothorax. Auch lokale Blutungen im Bereich des Stichkanals sowie pleurale Schmerzen sind hier zu nennen. Postinterventionell stellt die Drahtdislokation ein Risiko dar. Diese kann beim Transport des Patienten, bei der Umlagerung auf den OP-Tisch oder auch während des Eingriffs passieren, insgesamt ist das Risiko mit dem von uns vorgestellten System und einer Dislokationsrate von ca. 3\% jedoch geringer als bei anderen Systemen.

Die Intervention führten an unserer Klinik größtenteils erfahrene Fachärzte durch, jedoch erfolgten auch Markierungen durch Assistenzärzte unter Anleitung eines Facharztes ohne größere Komplikationen. Dies spricht ebenfalls für die Sicherheit und leichte Handhabung der Methode.

Als Limitation der Studie kann der retrospektive Charakter gelten, allerdings sind bezüglich der erhobenen Daten auch in einer prospektiven Studie keine anderen Werte zu erwarten. Zudem ist dies nach unserem Kenntnisstand die größte bislang veröffentlichte Studie zu diesem speziellen Markierungssystem, welche auch in einer retrospektiven Analyse die Sicherheit der Methode belegen kann.

Zusammenfassend lässt sich sagen, dass das vorgestellte Kombinationsverfahren bestehend aus präoperativer Drahtmarkierung und anschließender videothorakoskopischer Entfernung der markierten Herde eine wertvolle und sichere Methode für den Patienten darstellt. Die einfache Handhabung des verwendeten Systems, welche eine schnelle und treffsichere Markierung und eine stabile Fixation ermöglicht, sowie die geringe Komplikationsrate sprechen für eine breite Anwendung dieses Verfahrens zur Abklärung von peripher gelegenen pulmonalen Rundherden.

\section{Literatur}

1 Tan BB, Flaherty KR, Kazerooni AE et al. The solitary pulmonary nodule. Chest 2003; 123: 89-96

2 MacMahon H, Austin JHM, Gamsu G et al. Guidelines for management of small pulmonary nodules detected on CT scans: a statement from the Fleischner Society. Radiology 2005; 237: 395-400

3 Lewis RJ, Caccavale RJ, Sisler GE. Imaged thoracoscopic lung biopsy. Chest 1992; 102: 60-62

4 Kaiser LR, Shrager JB et al. Video-assisted thoracic surgery: the current state of the art. Am J Roentgenol 1995; 165: 1111-1117

5 Bernard $A$ The Thorax Group. Resection of pulmonary nodules using video-assisted thoracic surgery. Ann Thorac Surg 1996; 61: 202 - 204

6 Ciriaco P, Negri G, Puglisi A et al. Video-assisted thoracoscopic surgery for pulmonary nodules: rationale for preoperative computed tomography-guided hookwire localization. Eur J Cardiothorac Surg 2004; 25: 429-433

7 Chen YR, Yeow KM, Lee JY et al. CT-guided hook wire localization of subpleural lung lesions for video-assisted thoracoscopic surgery (VATS). J Formos Med Assoc 2007; 11: 911 -918

8 Partrick DA, Bensard DD, Teitelbaum DH et al. Successful thoracoscopic lung biopsy in children utilizing preoperative CT-guided localization. J Pediatr Surg 2002; 37: 970-973

9 Willekes L, Boutros C, Goldfarb MA. VATS intraoperative tattooing to facilitate solitary pulmonary nodule resection. J Cardiothorac Surg 2008; 3: 13 
10 Boni G, Bellina CR, Grosso M et al. Gamma probe-guided thoracoscopic surgery of small pulmonary nodules. Tumori 2000; 86: 364-366

11 Kawanaka K, Nomori H, Mori T et al. Marking of small pulmonary nodules before thoracoscopic resection: injection of lipiodol under CTfluoroscopic guidance. Acad Radiol 2009; 16: 39-45

12 Celik M, Halezeroglu S, Senol C et al. Video-assisted thoracoscopic surgery: experience with 341 cases. Eur J Cardiothorac Surg 1998; 14: $113-116$

13 Hirau S, Hamanaka Y, Mitsui $N$ et al. Role of video-assisted thoracic surgery for the diagnosis of indeterminate pulmonary nodule. Ann Thorac Cardiovasc Surg 2006; 12: 388 - 392

14 Belgers EH, Siebenga J, Bosch AM et al. Complete video-assisted thoracoscopic surgery lobectomy and its learning curve. A single center study introducing the technique in The Netherlands. Interact Cardiovasc Thorac Surg 2010; 10: 176-180

15 Daniel TM, Kern JA, Tribble CG et al. Thoracoscopic surgery for diseases of the lung and pleura. Effectiveness, changing indications, and limitations. Ann Surg 1993; 217: 566 - 574

16 Landreneau R, Mack M, Hazelrigg S et al. Prevalence of chronic pain after pulmonary resection by thoracotomy or video-assisted thoracic surgery. J Thorac Cardiovasc Surg 1994; 107: 1079-1086

17 Ferson PF, Landreneau RJ, Dowhing RD et al. Comparison of open versus thoracoscopic lung biopsy for diffuse infiltrative pulmonary disease. J Thorac Cardiovasc Surg 1993; 106: 194-199

18 Suzuki K, Nagai K, Yoshida J et al. Video-Assisted thoracoscopic surgery for small indeterminate pulmonary nodules: indications for preoperative marking. Chest 1999; 115: 563-568

19 Tamura M, Oda M, Fujimori $H$ et al. New indication for preoperative marking of small peripheral pulmonary nodules in thoracoscopic surgery. Interact CardioVasc Thorac Surg 2010; 11: 590-593
20 Kloeppel R, Friedrich T, Eichfeld U et al. CT-gesteuerte Lungenherdmarkierung vor minimal-invasiver Operation. Radiologe 2001; 41: 201 204

21 Eichfeld $U$, Dietrich A, Ott $R$ et al. Video-assisted thoracoscopic surgery for pulmonary nodules after computed tomography-guided marking with a spiral wire. Ann Thorac Surg 2005; 79: 313-317

22 Torre M, Ferraroli GM, Vanzulli A et al. A new safe and stable spiral wire needle for thoracoscopic resection of lung nodules. Chest 2004; 125 : $2289-2293$

23 Lenglinger FX, Schwarz CD, Artmann W. Localization of pulmonary nodules before thoracoscopic surgery: value of percutaneous staining with methylene blue. Am J Roentgenol 1994; 163: 297 - 300

24 Shah RM, Spirn PW, Salazar AM et al. Localization of peripheral pulmonary nodules forthoracoscopic excision: value of CT-guided wire placement. Am J Roentgenol 1993; 161: 279-283

25 Powell TI, Jangra D, Clifton JC et al. Fluoroscopically guided video-assisted thoracoscopic resection after computed tomography-guided localization using platinum microcoils. Ann Surg 2004; 240: 481 - 489

26 Mullan BF, Stanford W, Barnhart W et al. Lung Nodules: improved wire for CT-guided localization. Radiology 1999; 211: 561 - 565

27 Thaete FL, Peterson MS, Plunkett MB et al. Computed tomography-guided wire localization of pulmonary lesions before thoracoscopic resection: results in 101 cases. J Thorac Imag 1999; 14: 90-98

28 Yoshida Y, Inoh S, Murakawa T et al. Preoperative localization of small peripheral pulmonary nodules by percutaneous marking under computed tomography guidance. Interact CardioVasc Thorac Surg 2011; 13: $25-28$

29 Gonfiotti A, Davini F, Vaggelli L et al. Thoracoscopic localization techniques for patients with solitary pulmonary nodule: hookwire versus radio-guided surgery. Eur J Cardiothorac Surg 2007; 32: 843-847 


\section{Zusammenfassung}

Publikationspromotion zur Erlangung des akademischen Grades Dr. med.

Promotionstitel: $\quad$ CT-gestützte Drahtmarkierung vor videoassistierter thorakoskopischer OP von pulmonalen Rundherden - eine Auswertung von 184 Fällen

Eingereicht von: Marie-Kristin Schulze

Angefertigt am / in: Universität Leipzig / Medizinische Fakultät

Klinik und Poliklinik für Diagnostische und Interventionelle Radiologie

Betreut von: $\quad$ Prof. Dr. med. Thomas Kahn

OA Dr. med. Patrick Stumpp,

Klinik und Poliklinik für Diagnostische und Interventionelle Radiologie

Mai 2012

Diese Arbeit befasst sich mit der CT-gestützten Drahtmarkierung vor videoassistierter thorakoskopischer Operation von pulmonalen Rundherden, eine Variante der präoperativen Markierung zur Sicherstellung der intraoperativen Herdlokalisation. Die Zielsetzung bestand dabei in der Beurteilung der Wertigkeit dieser Markierungsmethode als radiologisch interventionelles Verfahren. Die Erfolgsrate, die Sicherheit der Intervention sowie aufgetretene Komplikationen wurden bei 184 durchgeführten Markierungen evaluiert.

Als Folge der sich ständig weiterentwickelnden bildgebenden Verfahren und der derzeit etablierten Lungen-CT in Spiraltechnik werden heutzutage weit mehr Lungenherde diagnostiziert als noch vor wenigen Jahren. Diese Entwicklungen geben Anlass zur Hoffnung, dass zukünftig mehr Karzinome bereits in sehr frühen Stadien entdeckt und therapiert werden können. Die Bewertung pulmonaler Rundherde unklarer Dignität bleibt jedoch ohne invasives Vorgehen mit der Gewinnung einer Diagnose bringenden Histologie schwierig. Diese stellt jedoch die Voraussetzung für eine adäquate Therapie dar. Hier kommt der videoassistierten thorakoskopischen Chirurgie eine entscheidende Rolle zu. Dieses minimalinvasive Verfahren stellt heute eine Alternative zur konventionellen offenen Thorakotomie dar, um pulmonale Herde zu resezieren. Vielfältige Vorteile dieser Methode lassen sich aufführen (Klöppel et al. 2001):

- komplikationsarme Durchführung 
- geringe Schmerzbelastung

- kosmetischer Nutzen

- reduzierte Krankenhausaufenthaltsdauer und Rekonvaleszenz

Als nachteilig sind hier die Bedingung der relativ peripheren Lage des zu resezierenden Herdes sowie die fehlende Möglichkeit der digitalen Austastung der Lunge während der videothorakoskopischen Exploration $\mathrm{zu}$ werten. Um dennoch eine sichere, komplikationsarme intraoperative Lokalisation und somit Resektion der pulmonalen Rundherde zu gewährleisten, welche bei einer endoskopischen Operation weder tast- noch sichtbar sind, haben sich vielfältige präoperative Markierungstechniken etabliert. Verschiedene Formen von Markierungsdrähten, wie Anker- und Hakendrähte (Ciriaco et al. 2004; Chen et al. 2007), Spiraldrähte oder ursprünglich für die Markierung suspekter Mamma-Befunde entwickelte Drähte (Mullan et al. 1999; Thaete et al. 1999) wurden verwendet. Weiterhin nutzt man Radionuklide (Boni et al. 2000), Lipiodol (Kawanaka et al. 2009) oder Methylenblau (Willekes et al. 2008) zur Anfärbung der Herde präoperativ. Jede Markierungsmethode zeigt ihre jeweiligen Vor- und Nachteile, ein Goldstandard für die präoperative Herdmarkierung lässt sich derzeit nicht benennen.

Wir verwendeten in unserer Einrichtung in den letzten zehn Jahren das Lungen-MarkerSystem der Firma Somatex ${ }^{\circledR}$ und fanden sehr gute Ergebnisse. Dieser eigens zu diesem Zweck entwickelte Spiraldraht weist vielfältige Vorteile gegenüber anderen Markierungstechniken auf: Neben einer leichten Handhabung des Systems lässt sich hier die stabile, aber dennoch sehr flexible Materialeigenschaft des Drahtes nennen. Durch die Spiralform lässt sich eine sichere Verankerung im weichen Lungengewebe erreichen, das biegsame extrathorakale Ende erlaubt dahingegen die äußere Fixierung und lässt intraoperativ eine dosierte Zugkraft-Anwendung zu. Die koaxiale Ladekanülentechnik ermöglicht eine Korrigierbarkeit des Drahtsystems. Auch das akzeptable Punktionsrisiko bei der Verwendung einer lediglich 18 Gauge dicken Nadel stellt einen Benefit der Methode dar (Kloeppel et al. 2001; Torre et al. 2004; Eichfeld et al. 2005).

In dieser Arbeit werden unseren bisherigen Erfahrungen bzw. Ergebnisse mit diesem Lungenmarkierungsdraht dargestellt. 
In dem Zeitraum von Oktober 1999 bis März 2010 wurden 184 CT-gestützte Drahtmarkierungen vorgenommen. Alle Patienten unterzogen sich unmittelbar anschließend der videothorakoskopischen Entfernung der pulmonalen Rundherde.

Circa ein Drittel der Herde (33,7\%) fielen als Zufallsbefunde während medizinischer Untersuchungen aus anderen Gründen auf, etwa die Hälfte $(51,1 \%)$ wurde im Rahmen von Nachuntersuchungen bei bekannter maligner Grunderkrankung entdeckt und 15,2\% der Befunde wurden während Staginguntersuchungen bei erst kürzlich diagnostizierter maligner Erkrankung detektiert. Das Durchschnittsalter der Patienten lag bei 58,1 Jahren. Rechtsseitig fanden sich 111 der Rundherde (davon $41 \mathrm{im} \mathrm{OL,} 20 \mathrm{im} \mathrm{ML,} 50 \mathrm{im} \mathrm{UL),} \mathrm{auf} \mathrm{die} \mathrm{linke} \mathrm{Seite}$ entfielen 73 Läsionen (davon 30 im OL, 43 im UL). Die Größe der Herde lag durchschnittlich bei 7,0 × 9,7 mm, die Entfernung zur Pleura betrug im Mittel 9,8 mm.

Die CT-gesteuerte Markierung erfolgte in Lokalanästhesie. Unter CT-gestützter Lagekontrolle wurde die Führungsnadel eingebracht. Nach Sicherung der richtigen Position der Nadel, wurde anschließend der Mandrin entfernt und durch eine Hohlnadel mit inliegendem Führungsdraht ersetzt. Der Draht, welcher nach Entfaltung seine ursprüngliche Spiralform annimmt, wurde dann vorgeschoben, wenn die korrekte Nadelposition möglichst nah am bzw. im Rundherd sichergestellt war.

In 181 Fällen $(98,4 \%)$ konnte der Markierungsdraht erfolgreich platziert und transthorakal fixiert werden. Eine Markierung musste aufgrund eines nicht beherrschbaren Pneumothorax abgebrochen werden, bei zwei Patienten kam es während der Markierung zu einer Drahtdislokation. Von den geringgradigen, methodisch nicht vermeidbaren Komplikationen standen erwartungsgemäß Mantelpneumothoraces (53,3\%) und perifokale Blutungen $(30,4 \%)$ im Vordergrund, bedurften jedoch keiner weiterführenden Therapie und stellen unseren Erachtens nach durchaus akzeptable Risiken dar. Ähnliche Komplikationsraten zeigen auch frühere Veröffentlichungen, Yoshida et al. (2011) berichten beispielsweise über Pneumothoraxraten von $49,1 \%$ sowie kleinere intrathorakale Blutungen in $29,8 \%$ der Fälle. Die mittlere Interventionsdauer lag in unserer Klinik bei 30,9 min und ist damit vergleichbar mit Interventionszeiten anderer Untersuchungen. Die Markierung mit Platin-Microcoils, genutzt von Powell und Kollegen (2004), dauerte im Mittel 42 min. Chen et al. (2007) führten Markierungen mit Hakendrähten im Durchschnitt in 30,4 min durch. 
Bis zum Beginn der VATS kam es lediglich bei 6 Patienten (3,3\%) zu Drahtdislokationen. In der Literatur wird über Dislokationsraten von bis zu $20 \%$ bei der Verwendung von MammaMarkierungsdrähten berichtet (Shah et al. 1993; Mullan et al. 1999; Thaete et al. 1999).

Die anschließende thorakoskopische Resektion der markierten Herde erfolgt über lediglich drei kleine Inzisionen von circa 1,5 cm Länge. Nach dem Ausklemmen der betroffenen Lungenseite von der Beatmung erfolgt die orientierende Thorakoskopie. Ist der gesuchte Herd anhand des Drahtverlaufes identifiziert, kann dieser nun durch leichten Zug am externen Drahtanteil in Richtung der Thoraxwand verlagert werden. Schließlich erfolgt die Resektion mittels Endo-linear-Cutter ${ }^{\circledR}$ (Ethicon, Norderstedt, Deutschland) sowie die Bergung des Rundherdes mit dem intrathorakal gelegenen Drahtabschnitt in einem Bergebeutel.

Eine makroskopisch vollständige Entfernung der markierten Herde gelang bei $98,4 \%$ der Patienten. In 29 Fällen (15,9\%) musste man aufgrund von intraoperativen Komplikationen (Adhäsionen, Blutungen, Drahtdislokationen) auf eine offene Thorakotomie umsteigen. Die Histologie ergab in 96 Fällen (52,5\%) einen benignen Befund, wobei es sich in der Mehrzahl der Fälle um anthrakotische oder fibrotische Lymphknoten (37,5\%) sowie entzündliche Geschehen $(15,6 \%)$ handelte. In 87 Fällen (47,5\%) zeigten sich maligne Erkrankungen, wobei insgesamt lediglich 21 Herde (11,5\%) einem primären Lungenkarzinom entsprachen.

Nach der klinischen Einführung der videoassistierten Thoraxchirurgie vor circa zwanzig Jahren hat diese die offene Thorakotomie bei ausschließlich diagnostischer Indikation weitgehend abgelöst und macht in thoraxchirurgischen Zentren heutzutage etwa $20-25 \%$ aller Eingriffe aus (Dienemann et al. 2003). Die sichere Diagnosestellung und komplette Entfernung des Rundherdes bei nur relativ geringer Belastung für den Patienten stellen einen großen Benefit der Methode dar. Da bei einer endoskopischen Operation jedoch sehr kleine Rundherde oftmals nicht sichtbar sind, und die Möglichkeit der manuellen Austastung des Lungengewebes nicht gegeben ist, stellt sich die intraoperative Lokalisation in vielen Fällen schwierig dar. Um also ein komplikationsarmes und schnelles Auffinden des gesuchten Herdes während der VATS zu ermöglichen, hat sich die präoperative CT-gesteuerte Drahtmarkierung von Rundherden etabliert und lässt sich unseren Erfahrungen nach zu urteilen als komplikationsarme und treffsichere Markierungsvariante empfehlen. Die 
schnelle und leichte Handhabung des Systems, die geringe Komplikationsrate sowie die stabile Fixation des Herdes im weichen Lungengewebe sprechen für eine präoperative Markierung vor allem kleiner, subpleural gelegener Lungenherde mit diesem speziellen Lungen-Markierungs-System. 


\section{Literatur}

Belgers EH, Siebenga J, Bosch AM et al. Complete video-assisted thoracoscopic surgery lobectomy and its learning curve. A single center study introducing the technique in The Netherlands. Interact Cardiovasc Thorac Surg 2010; 10: 176-180

Bernard A The Thorax Group. Resection of pulmonary nodules using video-assisted thoracic surgery. Ann Thorac Surg 1996; 61: 202-204

Boni G, Bellina CR, Grosso $M$ et al. Gamma probe-guided thoracoscopic surgery of small pulmonary nodules. Tumori 2000; 86: 364-366

Braimbridge MV. The history of thoracoscopic surgery. Ann Thorac Surg 1993; 56: 610-614

Celik M, Halezeroglu S, Senol C et al. Video-assisted thoracoscopic surgery: experience with 341 cases. Eur J Cardiothorac Surg 1998; 14: 113-116

Chella A, Lucchi M, Ambrogi MC et al. A pilot study of the role of TC-99 radionuclide in localization of pulmonary nodular lesions for thoracoscopic resection. Eur J Cardiothorac Surg 2000; 18: 17-21

Chen YR, Yeow KM, Lee JY et al. CT-guided hook wire localization of subpleural lung lesions for video-assisted thoracoscopic surgery (VATS). J Formos Med Assoc 2007; 11: 911-918

Ciriaco P, Negri G, Puglisi A et al. Video-assisted thoracoscopic surgery for pulmonary nodules: rationale for preoperative computed tomography-guided hookwire localization. Eur J Cardiothorac Surg 2004; 25: 429-433

Daniel TM, Kern JA, Tribble CG et al. Thoracoscopic surgery for diseases of the lung and pleura. Effectiveness, changing indications, and limitations. Ann Surg 1993; 217: 566-574

Dienemann H, Hoffmann H. Chancen der endoskopischen Thoraxchirurgie und ihre Grenzen. Chirurg 2003; 74: 324-332

Dohrmann P, Böhle AS, Kurdow R et al. Videoassistierte Thorakoskopie zur Behandlung maligner Pleuraergüsse: Thorakoskopische Talkumpleurodese und pleuroperitoneale Shuntanlage - retrospektive Untersuchung von 180 Eingriffen. Schlesw Holst Ärztebl 2010; 10: $27-31$ 
Eckersberger F. Guidelines in minimal invasive surgery in oncologic thoracic surgery. Langenbecks Arch Chir 1997; 114: 246-250

Eichfeld $U$, Dietrich A, Ott $R$ et al. Video-assisted thoracoscopic surgery for pulmonary nodules after computed tomography-guided marking with a spiral wire. Ann Thorac Surg 2005; 79: 313-317

Ferson PF, Landreneau RJ, Dowhing RD et al. Comparison of open versus thoracoscopic lung biopsy for diffuse infiltrative pulmonary disease. J Thorac Cardiovasc Surg 1993; 106: 194199

Fischbach F, Knollmann F, Griesshaber V et al. Detection of pulmonary nodules by multislice computed tomography: improved detection rate with reduced slice thickness. Eur Radiol 2003; 13: 2378-2383

Gatzemeier M. Die Bedeutung der Zytologie in der bronchologischen Diagnostik des Bronchialkarzinoms. Medizinische Dissertation. Hamburg. 2002

Gonfiotti A, Davini F, Vaggelli L et al. Thoracoscopic localization techniques for patients with solitary pulmonary nodule: hookwire versus radio-guided surgery. Eur J Cardiothorac Surg 2007; 32: 843-847

Gurney JW. Determining the likelihood of malignancy in solitary pulmonary nodules with Bayesian analysis. Part I. Theory. Radiology 1993; 186: 405-413

Hirau S, Hamanaka Y, Mitsui N et al. Role of video-assisted thoracic surgery for the diagnosis of indeterminate pulmonary nodule. Ann Thorac Cardiovasc Surg 2006; 12: 388-392

Hoffmann H, Dienemann H. Der pulmonale Rundherd: Prinzipien der Diagnostik. Dtsch Arztebl 2000; 97: 907-912

Inderbitzi R, Furrer M, Ris HB. Thorakoskopische Eingriffe. Zentralbl Chir 1993; 118: 322-328 Jacobaeus HC. Die Thorakoskopie und ihre praktische Bedeutung. Ergeb Ges Med (Berlin) 1925; 8: 112-166

Jacobaeus HC. Über die Möglichkeit, die Zystoskopie bei Untersuchungen seröser Hohlungen anzuwenden. Münch Med Wochenschr 1910; 57: 2090-2092 
Jimenez MF. Prospective study on video-assisted thoracoscopic surgery in the resection of pulmonary nodules: 209 cases from the Spanish Video-Assisted Thoracic Surgery Study Group. Eur J Cardiothorac Surg 2001; 19: 562-565

Kaiser LR, Shrager JB et al. Video-assisted thoracic surgery: the current state of the art. Am J Roentgenol 1995; 165: 1111-1117

Kawanaka K, Nomori H, Ikeda $\mathrm{K}$ et al. Marking of small pulmonary nodules before thoracoscopic resection: injection of lipiodol under CT-fluoroscopic guidance. Acad Radiol 2009; 16: 39-45.

Kim SJ, Lee HS, Kim HS et al. Outcome of video-assisted thoracoscopic surgery for spontaneous secondary pneumothorax. Korean J Thorac Cardiovasc Surg 2011; 44: 225-228

Kloeppel R, Friedrich T, Eichfeld U et al. CT-gesteuerte Lungenherdmarkierung vor minimalinvasiver Operation. Radiologe 2001; 41: 201-204

Kruger K, Eyl G, Morgenroth C et al. Success and complication rate of CT-guided marking of pulmonary nodules with coil wires for video-assisted thoracoscopic surgery (VATS). Rofo 2006; 178: 1250-1254.

Landreneau R, Hazelrigg S, Mack M et al. Postoperative pain-related morbidity: videoassisted thoracic surgery versus thoracotomy. Ann Thorac Surg 1993; 56: 1285-1289

Landreneau R, Mack M, Hazelrigg $S$ et al. Prevalence of chronic pain after pulmonary resection by thoracotomy or video-assisted thoracic surgery. J Thorac Cardiovasc Surg 1994; 107: $1079-1086$

Lenglinger FX, Schwarz CD, Artmann W. Localization of pulmonary nodules before thoracoscopic surgery: value of percutaneous staining with methylene blue. Am J Roentgenol 1994; 163: 297-300

Lewis RJ, Caccavale RJ, Sisler GE. Imaged thoracoscopic lung biopsy. Chest 1992; 102: 60-62

Lillington GA, Caskey $\mathrm{Cl}$. Evaluation and management of solitary and multiple pulmonary nodules. Clin Chest Med 1993; 14: 111-119 
Mack MJ, Hazelrigg SR, Landreneau RJ et al.. Thoracoscopy for the diagnosis of the indeterminate solitary pulmonary nodule. Ann Thorac Surg 1993; 56: 825-830

MacMahon H, Austin JHM, Gamsu G et al. Guidelines for management of small pulmonary nodules detected on CT scans: a statement from the Fleischner Society. Radiology 2005; 237: $395-400$

Mullan BF, Stanford W, Barnhart W et al. Lung Nodules: improved wire for CT-guided localization. Radiology 1999; 211: 561-565

Nomori $\mathrm{H}$, Horio $\mathrm{H}$, Naruke $\mathrm{T}$ et al. Fluoroscopy-assisted thoracoscopic resection of lung nodules marked with lipiodol. Ann Thorac Surg 2002; 74: 170-173

Ost D, Fein AM, Feinsilver SH. The solitary pulmonary nodule. N Engl J Med 2003; 348: 25352542

Partrick DA, Bensard DD, Teitelbaum DH et al. Successful thoracoscopic lung biopsy in children utilizing preoperative CT-guided localization. J Pediatr Surg 2002; 37: 970-973

Pirker M. Wertigkeit der video-assistierten Thorakoskopie in der Behandlung des Spontanpneumothorax und anderer thoraxchirurgisch therapierbarer Krankheitsbilder. Medizinische Dissertation. München. 2005

Powell TI, Jangra D, Clifton JC et al. Fluoroscopically guided video-assisted thoracoscopic resection after computed tomography-guided localization using platinum microcoils. Ann Surg 2004; 240: 481-489

Reynolds W. The first laparoscopic cholecystectomy. JSLS 2001; 5: 89-94

Rieger R, Wayand W. Video-assisted thoracoscopy in diagnosis and therapy of intrathoracic diseases. Zentralbl Chir 1997; 122: 1065-1071

Sedrakyan A, van der Meulen J, Lewsey J et al. Video assisted thoracic surgery for treatment of pneumothorax and lung resections: systematic review of randomised clinical trials. BMJ 2004; 329: 1008

Semm K. Endoscopic appendectomy. Endoscopy 1983; 15: 59-64 
Shah RM, Spirn PW, Salazar AM et al. Localization of peripheral pulmonary nodules for thoracoscopic excision: value of CT-guided wire placement. Am J Roentgenol 1993; 161: 279283

Shiau MC, Bonavita J, Naidich DP. Adenocarcinoma of the lung: current concepts in radiologic diagnosis and management. Curr Opin Pulm Med. 2007; 13: 261-266

Siegelman SS, Khouri NF, Leo FP et al. Solitary pulmonary nodules: CT assessment. Radiology 1986; 160: 307-312

Solli P, Spaggiari L. Indications and development of video-assisted thoracic surgery in the treatment of lung cancer. The Oncologist 2007; 12: 1205-1214

Suzuki K, Nagai K, Yoshida J et al. Prognostic factors in clinical stage I nonsmall cell lung cancer. Ann Thorac Surg 1999; 67: 927-932

Suzuki K, Nagai K, Yoshida J et al. Video-assisted thoracoscopic surgery for small indeterminate pulmonary nodules: indications for preoperative marking. Chest 1999; 115: $563-568$

Tamura $\mathrm{M}$, Oda $\mathrm{M}$, Fujimori $\mathrm{H}$ et al. New indication for preoperative marking of small peripheral pulmonary nodules in thoracoscopic surgery. Interact CardioVasc Thorac Surg 2010; 11: 590-593

Tan BB, Flaherty KR, Kazerooni AE et al. The solitary pulmonary nodule. Chest 2003; 123: 8996

Thaete FL, Peterson MS, Plunkett MB et al. Computed tomography-guided wire localization of pulmonary lesions before thoracoscopic resection: results in 101 cases. J Thorac Imag 1999; 14: 90-98

Thomas L. Klinische Aussagekraft und Komplikationen der CT-gesteuerten transthorakalen Biopsie. Vergleich dreier Biopsiesysteme. Medizinische Dissertation. Bonn. 2005

Torre M, Ferraroli GM, Vanzulli A et al. A new safe and stable spiral wire needle for thoracoscopic resection of lung nodules. Chest 2004; 125: 2289-2293 
Watanabe K, Nomori $\mathrm{H}$, Ohtsuka $\mathrm{T}$ et al. Usefulness and complications of computed tomography-guided lipiodol marking for fluoroscopy-assisted thoracoscopic resection of small pulmonary nodules: Experience with 174 nodules. J Thorac Cardiovasc Surg 2006; 132: $320-324$

Wicky S, Mayor B, Cuttat JF et al. CT- guided localizations of pulmonary nodules with methylene blue injections for thoracoscopic resections. Chest 1994; 106: 1326-1328

Willekes L, Boutros C, Goldfarb MA. VATS intraoperative tattooing to facilitate solitary pulmonary nodule resection. J Cardiothorac Surg 2008; 3: 13

Yim AP, Liu HP. Complications and failures of video-assisted thoracic surgery: experience from two centers in Asia. Ann Thorac Surg 1996; 61: 538-541

Yoshida Y, Inoh S, Murakawa T et al. Preoperative localization of small peripheral pulmonary nodules by percutaneous marking under computed tomography guidance. Interact CardioVasc Thorac Surg 2011; 13: 25-28 